\title{
A DFT perspective on the structures and electronic spectra of the orange and blue isomers of photochromic dithizonatophenylmercury(II)
}

\section{Supporting information}



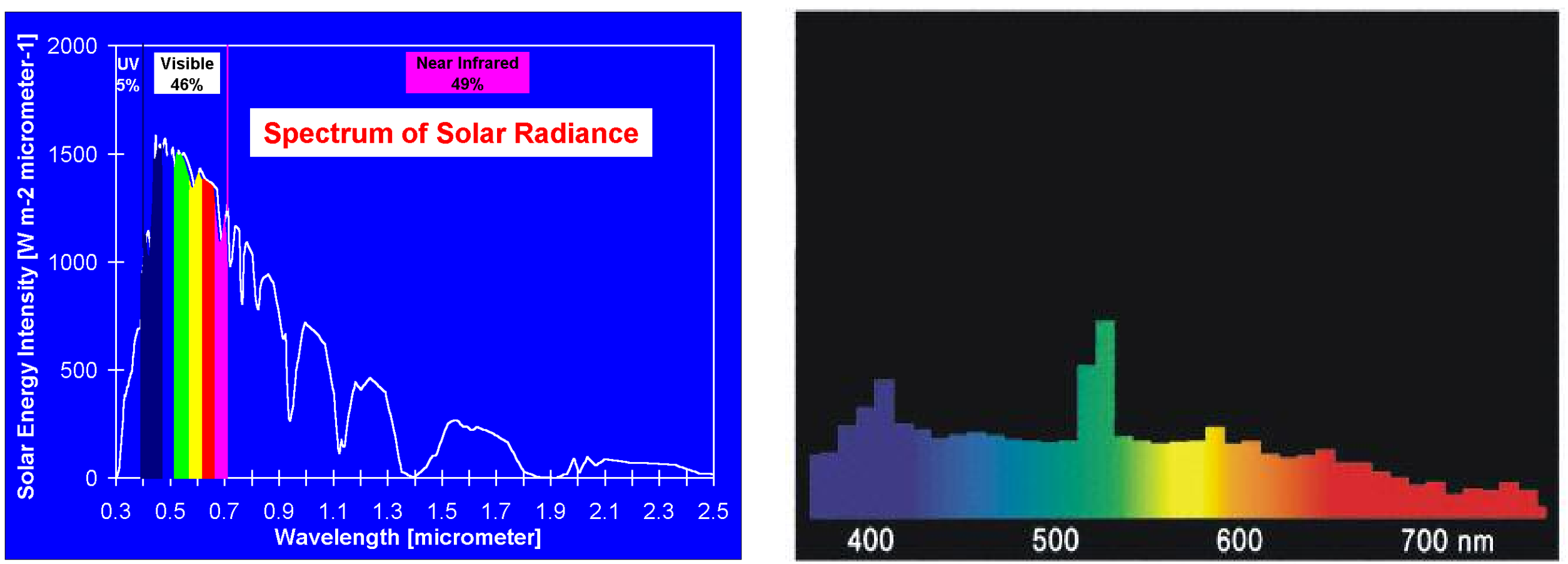

a

Figure S1. (a) Solar Spectral Distribution (Cornell University), and (b) spectral distribution of Osram Powerstar HQI-T 400W/D lamp (Osram). 


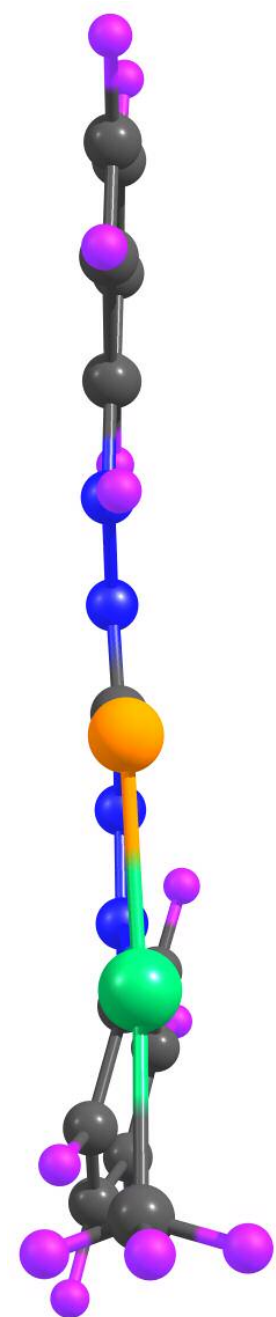

a: X-ray

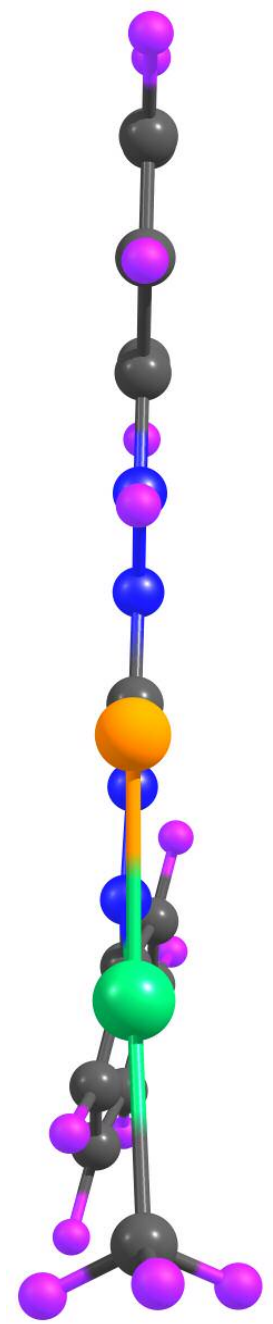

b: ADF/PW91

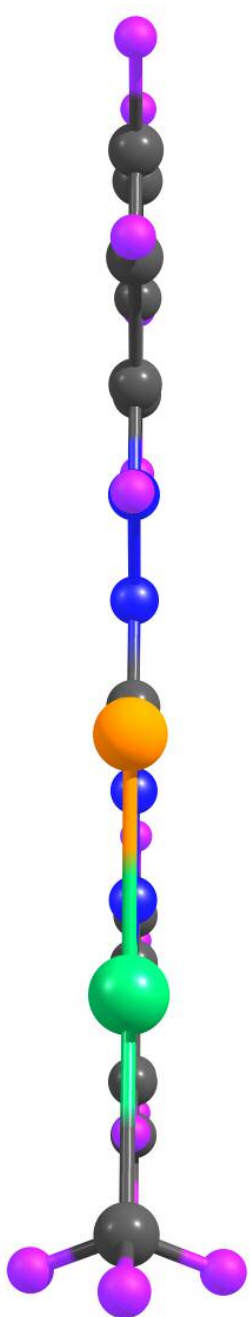

c: G03/B3LYP

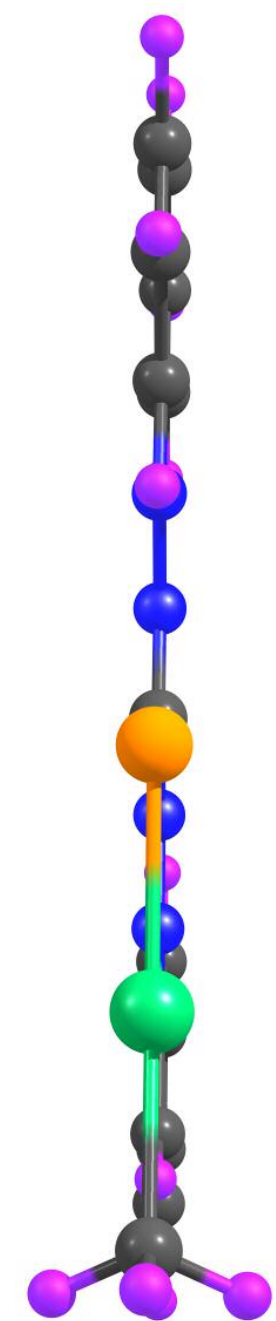

d: G03/HF

Figure S2. Side perspectives of the orange isomer of MeHgHDz, comparing the (a) X-Ray determined structure to (b) ADF (PW91), (c) G03 (B3LYP) and (d) G03/HF calculated geometries. (Green - Hg, orange - S, blue - N, violet - H, grey - C). Calculations at the Hartree-Fock levels of theory used the SDD (Stuttgart/Dresden effective core potential) basis set. 
Table S1. Selected bond lengths and angles of mercury complexes, also giving RMSD values and comparing (differences) computed data with crystallographic data.

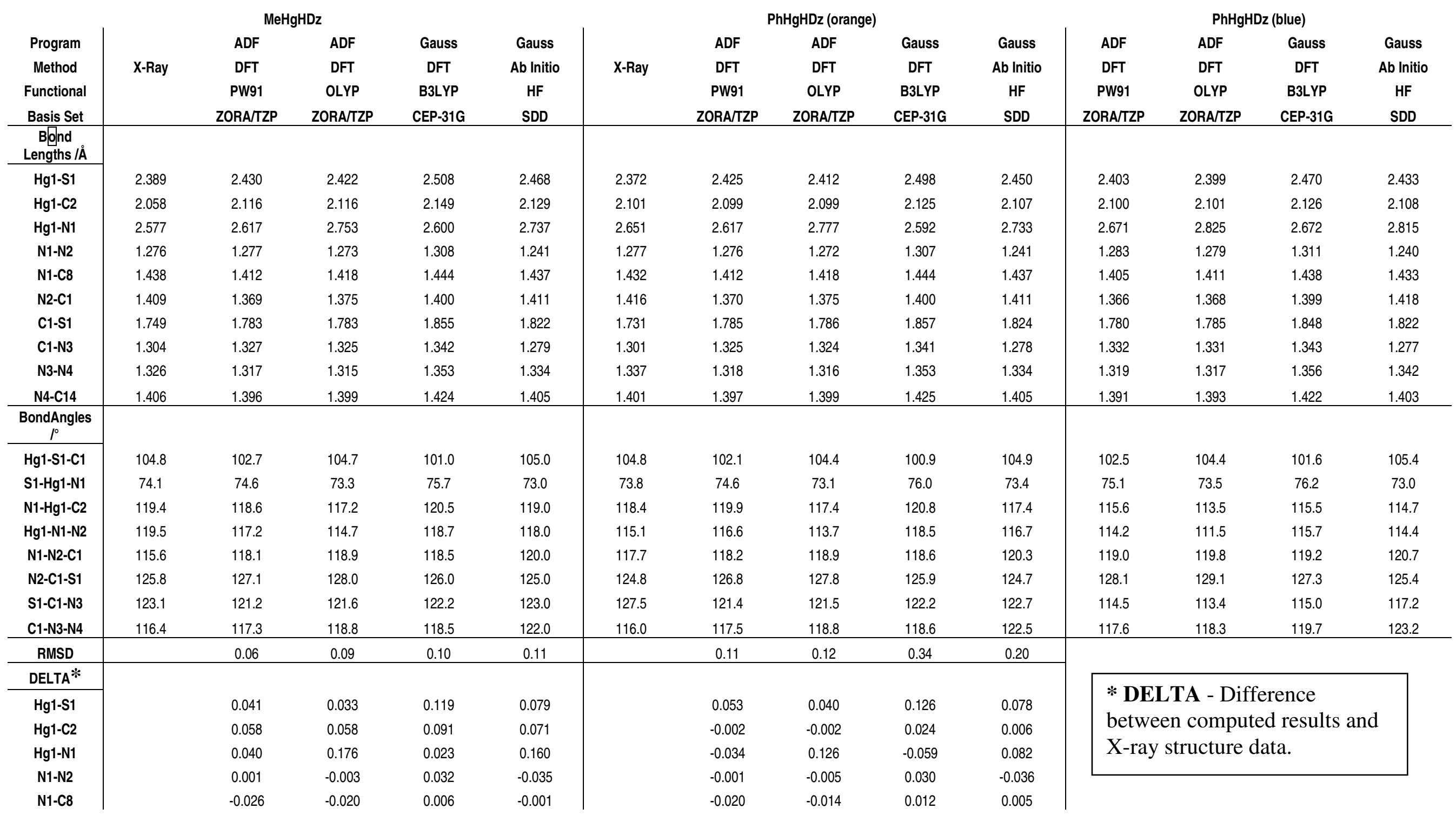




\begin{tabular}{|c|c|c|c|c|c|c|c|c|}
\hline $\mathrm{N} 2-\mathrm{C} 1$ & -0.040 & -0.034 & -0.009 & 0.002 & -0.046 & -0.041 & -0.016 & -0.005 \\
\hline C1-S1 & 0.034 & 0.034 & 0.106 & 0.073 & 0.054 & 0.055 & 0.126 & 0.093 \\
\hline C1-N3 & 0.023 & 0.021 & 0.038 & -0.025 & 0.024 & 0.023 & 0.040 & -0.023 \\
\hline N3-N4 & -0.009 & -0.011 & 0.027 & 0.008 & -0.019 & -0.021 & 0.016 & -0.003 \\
\hline $\mathrm{N} 4-\mathrm{C} 14$ & -0.010 & -0.007 & 0.018 & -0.001 & -0.004 & -0.002 & 0.024 & 0.004 \\
\hline \multicolumn{9}{|l|}{ DELTA } \\
\hline Hg1-S1-C1 & -2.100 & -0.100 & -3.800 & 0.200 & -2.700 & -0.400 & -3.900 & 0.100 \\
\hline S1-Hg1-N1 & 0.500 & -0.800 & 1.600 & -1.100 & 0.800 & -0.700 & 2.200 & -0.400 \\
\hline N1-Hg1-C2 & -0.800 & -2.200 & 1.100 & -0.400 & 1.500 & -1.000 & 2.400 & -1.000 \\
\hline Hg1-N1-N2 & -2.300 & -4.800 & -0.800 & -1.500 & 1.500 & -1.400 & 3.400 & 1.600 \\
\hline N1-N2-C1 & 2.500 & 3.300 & 2.900 & 4.400 & 0.500 & 1.200 & 0.900 & 2.600 \\
\hline N2-C1-S1 & 1.300 & 2.200 & 0.200 & -0.800 & 2.000 & 3.000 & 1.100 & -0.100 \\
\hline S1-C1-N3 & -1.900 & -1.500 & -0.900 & -0.100 & -6.100 & -6.000 & -5.300 & -4.800 \\
\hline C1-N3-N4 & 0.900 & 2.400 & 2.100 & 5.600 & 1.500 & 2.800 & 2.600 & 6.500 \\
\hline
\end{tabular}

\begin{tabular}{|c|c|c|c|c|c|c|c|c|c|c|c|c|c|c|c|}
\hline \multicolumn{6}{|c|}{ MeHg(cptsc) } & \multicolumn{5}{|c|}{ PhHg(cptsc) } & \multicolumn{5}{|c|}{$\mathrm{PhHg}(\mathrm{ptsc})$} \\
\hline Program & & ADF & ADF & Gaussian & Gaussian & & ADF & ADF & Gaussian & Gaussian & & ADF & ADF & Gaussian & Gaussian \\
\hline Method & X-Ray & DFT & DFT & DFT & Ab Initio & X-Ray & DFT & DFT & DFT & Ab Initio & X-Ray & DFT & DFT & DFT & Ab Initio \\
\hline Functional & & PW91 & OLYP & B3LYP & HF & & PW91 & OLYP & B3LYP & $\mathrm{HF}$ & & PW91 & OLYP & B3LYP & $\mathrm{HF}$ \\
\hline \multicolumn{16}{|l|}{$\begin{array}{c}\text { Bond } \\
\text { Lengths / }\end{array}$} \\
\hline Hg1-S1 & 2.380 & 2.427 & 2.422 & 2.507 & 2.488 & 2.382 & 2.422 & 2.414 & 2.497 & 2.471 & 2.377 & 2.427 & 2.423 & 2.509 & 2.482 \\
\hline $\mathrm{Hg} 1-\mathrm{C} 2$ & 2.088 & 2.118 & 2.117 & 2.151 & 2.135 & 2.063 & 2.101 & 2.101 & 2.128 & 2.115 & 2.048 & 2.096 & 2.098 & 2.124 & 2.111 \\
\hline N1-N2 & 1.387 & 1.375 & 1.373 & 1.427 & 1.402 & 1.404 & 1.375 & 1.373 & 1.427 & 1.403 & 1.400 & 1.371 & 1.367 & 1.423 & 1.396 \\
\hline N1-C8 & 1.259 & 1.289 & 1.292 & 1.312 & 1.275 & 1.264 & 1.291 & 1.292 & 1.312 & 1.276 & 1.283 & 1.308 & 1.308 & 1.329 & 1.284 \\
\hline $\mathrm{N} 2-\mathrm{C} 1$ & 1.299 & 1.307 & 1.304 & 1.329 & 1.290 & 1.302 & 1.306 & 1.303 & 1.328 & 1.289 & 1.304 & 1.313 & 1.311 & 1.335 & 1.296 \\
\hline C1-S1 & 1.753 & 1.779 & 1.779 & 1.849 & 1.815 & 1.751 & 1.780 & 1.780 & 1.851 & 1.817 & 1.742 & 1.770 & 1.771 & 1.841 & 1.812 \\
\hline C1-N3 & 1.350 & 1.378 & 1.384 & 1.388 & 1.365 & 1.349 & 1.378 & 1.384 & 1.387 & 1.365 & 1.361 & 1.376 & 1.382 & 1.386 & 1.362 \\
\hline \multicolumn{16}{|l|}{$\begin{array}{c}\text { BondAngles } \\
1^{\circ}\end{array}$} \\
\hline $\mathrm{Hg} 1-\mathrm{S} 1-\mathrm{C} 1$ & 103.0 & 101.4 & 103.1 & 99.9 & 101.3 & 101.4 & 101.3 & 103.0 & 99.7 & 101.1 & 102.4 & 101.2 & 102.4 & 99.5 & 100.9 \\
\hline $\mathrm{N} 1-\mathrm{Hg} 1-\mathrm{C} 2$ & 117.0 & 116.8 & 116.7 & 119.1 & 119.5 & 120.3 & 118.9 & 117.6 & 119.6 & 119.0 & 118.4 & 116.4 & 116.2 & 118.2 & 117.5 \\
\hline Hg1-N1-N2 & 117.0 & 115.3 & 112.7 & 117.2 & 116.4 & 115.5 & 115.2 & 112.7 & 117.2 & 116.0 & 117.5 & 116.3 & 114.7 & 118.7 & 117.8 \\
\hline N1-N2-C1 & 114.0 & 116.3 & 117.0 & 116.1 & 117.8 & 114.4 & 116.4 & 117.2 & 116.1 & 117.9 & 113.4 & 116.1 & 116.4 & 115.4 & 116.9 \\
\hline N2-C1-S1 & 130.0 & 130.9 & 131.8 & 130.0 & 129.1 & 129.2 & 130.8 & 131.7 & 129.9 & 129.0 & 130.5 & 130.3 & 131.1 & 129.4 & 128.7 \\
\hline S1-C1-N3 & 111.0 & 112.8 & 112.2 & 113.1 & 113.4 & 114.0 & 112.8 & 112.2 & 113.1 & 113.3 & 112.3 & 113.0 & 112.6 & 113.4 & 113.4 \\
\hline
\end{tabular}




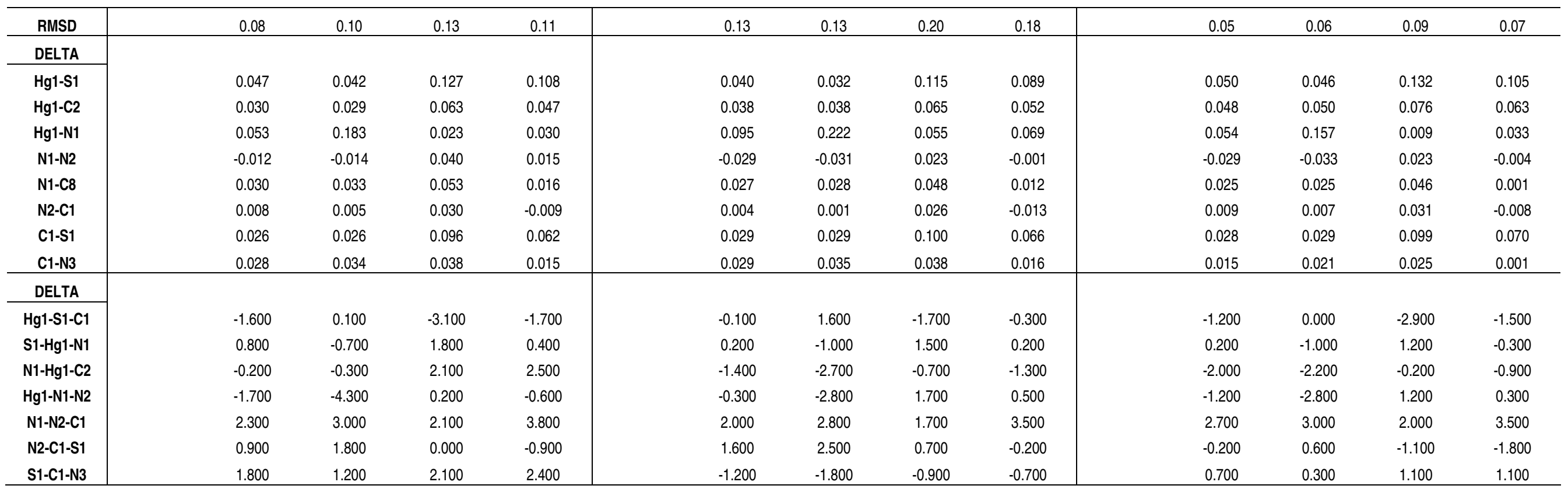

Table S2. Solvent effect on the geometry (bond lengths in $\AA$ and RMSD from crystallographic data) of the different isomers of PhHgHDz calculated with

ADF/PW91/ZORA/TZP, using the COSMO model.

PhHgHDz (orange)

\begin{tabular}{|c|c|c|c|c|c|c|c|c|c|c|c|c|c|c|c|c|}
\hline \multirow[b]{2}{*}{$\begin{array}{c}\text { Solvent } \\
\varepsilon \\
\end{array}$} & \multicolumn{6}{|c|}{ PhHgHDz (orange) } & \multicolumn{5}{|c|}{ PhHgHDz (blue) - N4H } & \multicolumn{5}{|c|}{ PhHgHDz (blue) - N2H } \\
\hline & X-ray & $\begin{array}{c}\text { vacuum } \\
0 \\
\end{array}$ & $\begin{array}{c}\text { hexane } \\
1.88 \\
\end{array}$ & $\begin{array}{c}\text { DCM } \\
8.9 \\
\end{array}$ & $\begin{array}{c}\text { methanol } \\
32.6 \\
\end{array}$ & $\begin{array}{l}\text { water } \\
78.39 \\
\end{array}$ & $\begin{array}{c}\text { vacuum } \\
0 \\
\end{array}$ & $\begin{array}{c}\text { hexane } \\
1.88 \\
\end{array}$ & $\begin{array}{c}\text { DCM } \\
8.9 \\
\end{array}$ & $\begin{array}{c}\text { methanol } \\
32.6 \\
\end{array}$ & $\begin{array}{l}\text { water } \\
78.39 \\
\end{array}$ & $\begin{array}{c}\text { vacuum } \\
0 \\
\end{array}$ & $\begin{array}{c}\text { hexane } \\
1.88 \\
\end{array}$ & $\begin{array}{c}\text { DCM } \\
8.9 \\
\end{array}$ & $\begin{array}{c}\text { methanol } \\
32.6 \\
\end{array}$ & $\begin{array}{l}\text { water } \\
78.39 \\
\end{array}$ \\
\hline Hg1-S1 & 2.372 & 2.425 & 2.426 & 2.426 & 2.428 & 2.430 & 2.403 & 2.403 & 2.407 & 2.408 & 2.410 & 2.427 & 2.427 & 2.427 & 2.430 & 2.431 \\
\hline Hg1-N1 & 2.651 & 2.617 & 2.618 & 2.618 & 2.623 & 2.632 & 2.671 & 2.671 & 2.674 & 2.676 & 2.676 & 2.631 & 2.631 & 2.633 & 2.641 & 2.648 \\
\hline N1-N2 & 1.277 & 1.276 & 1.276 & 1.276 & 1.277 & 1.277 & 1.283 & 1.283 & 1.283 & 1.283 & 1.283 & 1.324 & 1.324 & 1.324 & 1.323 & 1.322 \\
\hline N1-C8 & 1.432 & 1.412 & 1.412 & 1.412 & 1.412 & 1.412 & 1.405 & 1.405 & 1.405 & 1.405 & 1.406 & 1.390 & 1.390 & 1.390 & 1.390 & 1.390 \\
\hline N2-C1 & 1.416 & 1.370 & 1.369 & 1.369 & 1.369 & 1.370 & 1.366 & 1.365 & 1.364 & 1.363 & 1.362 & 1.337 & 1.337 & 1.337 & 1.338 & 1.337 \\
\hline C1-S1 & 1.731 & 1.785 & 1.785 & 1.785 & 1.786 & 1.786 & 1.780 & 1.781 & 1.785 & 1.786 & 1.787 & 1.741 & 1.742 & 1.742 & 1.744 & 1.745 \\
\hline C1-N3 & 1.301 & 1.325 & 1.326 & 1.325 & 1.326 & 1.326 & 1.332 & 1.332 & 1.331 & 1.331 & 1.331 & 1.377 & 1.377 & 1.377 & 1.375 & 1.374 \\
\hline N3-N4 & 1.337 & 1.318 & 1.318 & 1.318 & 1.317 & 1.317 & 1.319 & 1.319 & 1.319 & 1.318 & 1.318 & 1.289 & 1.289 & 1.289 & 1.291 & 1.291 \\
\hline N4-C14 & 1.401 & 1.397 & 1.397 & 1.379 & 1.398 & 1.398 & 1.391 & 1.391 & 1.392 & 1.392 & 1.392 & 1.398 & 1.398 & 1.398 & 1.398 & 1.398 \\
\hline RMSD & & 0.11 & 0.11 & 0.11 & 0.11 & 0.11 & & & & & & & & & & \\
\hline
\end{tabular}


Table S3. Effect on the geometry (bond lengths in $\AA$ and RMSD from crystallographic data) of the orange isomer of PhHgHDz calculated with ADF/PW91/ZORA/TZP, when taking relativistic effects into account.

\begin{tabular}{|c|lll|}
\multicolumn{1}{l}{} & X-ray & relativistic & non-relativistic \\
Hg1-S1 & 2.372 & 2.425 & 2.538 \\
Hg1-C2 & 2.101 & 2.099 & 2.191 \\
Hg1-N1 & 2.651 & 2.617 & 2.501 \\
N1-N2 & 1.277 & 1.276 & 1.280 \\
N1-C8 & 1.432 & 1.412 & 1.412 \\
N2-C1 & 1.416 & 1.370 & 1.367 \\
C1-S1 & 1.731 & 1.785 & 1.775 \\
C1-N3 & 1.301 & 1.325 & 1.331 \\
N3-N4 & 1.337 & 1.318 & 1.314 \\
N4-C14 & 1.401 & 1.397 & 1.397 \\
\hline RMSD & & 0.11 & 0.14 \\
\hline
\end{tabular}

Table S4. Solvent effect on the energetics (in $\mathrm{kJ} \cdot \mathrm{mol}^{-1}$ ) of the different isomers of PhHgHDz calculated with ADF/PW91/ZORA/TZP, using the COSMO model. Energies are compared to that of the orange isomer, to which a relative energy of $0 \mathrm{~kJ} \mathrm{~mol}^{-1}$ was assigned.

\begin{tabular}{c|ccccc} 
& Vacuum & Hexane & DCM & Methanol & Water \\
$\boldsymbol{\varepsilon}$ & $\mathbf{1}$ & $\mathbf{1 . 8 8}$ & $\mathbf{8 . 9}$ & $\mathbf{3 2 . 6}$ & $\mathbf{7 8 . 3 4}$ \\
\hline Structure N4H & 24.4 & 24.4 & 23.5 & 22.6 & 22.6 \\
Structure N2H & 60.1 & 60.5 & 61.6 & 61.6 & 61.6 \\
\hline
\end{tabular}


Table S5. Selected excitation energies (eV), wavelength ( $\mathrm{nm})$, oscillator strength (in a.u.) and main transitions for the allowed excitations of $\mathrm{PhHgHDz}$ (blue, $\mathrm{N} 4(\mathrm{H})$ ), $\mathrm{PhHgHDz}$ (orange) and $\mathrm{PhHgHDz}$ (blue, $\mathrm{N} 2(\mathrm{H})$ ).

\begin{tabular}{|c|c|c|c|c|c|c|c|c|}
\hline \multicolumn{9}{|c|}{ Gaussian/B3LYP/CEP-31G } \\
\hline & State & $\begin{array}{c}\text { Energy } \\
/ \mathrm{eV}\end{array}$ & $\begin{array}{c}\text { Wavelength } \\
\text { / nm }\end{array}$ & $\begin{array}{l}\text { Oscillator } \\
\text { Strength / }\end{array}$ & \multicolumn{4}{|c|}{$\begin{array}{c}\text { Transitions; } \\
\%\end{array}$} \\
\hline \multirow[t]{10}{*}{ BLUE N4 } & $\mathrm{S}_{1}$ & 1.99 & 622.7 & 0.298 & HOMO & $->$ & LUMO & $57.9 \%$ \\
\hline & & & & & HOMO-1 & $->$ & LUMO & $15.3 \%$ \\
\hline & $\mathrm{S}_{3}$ & 2.87 & 432.0 & 0.291 & HOMO-1 & $->$ & LUMO & $60.6 \%$ \\
\hline & $\mathrm{S}_{9}$ & 3.71 & 334.1 & 0.184 & HOMO-7 & $->$ & LUMO & $57.0 \%$ \\
\hline & & & & & HOMO-8 & $->$ & LUMO & $25.1 \%$ \\
\hline & $\mathrm{S}_{13}$ & 4.31 & 287.6 & 0.082 & HOMO & $->$ & LUMO+3 & $61.7 \%$ \\
\hline & & & & & HOMO-10 & $->$ & LUMO & $12.0 \%$ \\
\hline & $\mathrm{S}_{14}$ & 4.39 & 282.5 & 0.083 & HOMO & $->$ & LUMO+4 & $53.3 \%$ \\
\hline & & & & & HOMO & $->$ & LUMO+5 & $30.7 \%$ \\
\hline & & & & & HOMO-11 & $->$ & LUMO & $12.5 \%$ \\
\hline \multirow[t]{10}{*}{ ORANGE } & $\mathrm{S}_{1}$ & 2.61 & 474.4 & 0.566 & HOMO & $->$ & LUMO & $52.9 \%$ \\
\hline & & & & & HOMO-5 & $->$ & LUMO & $10.2 \%$ \\
\hline & $\mathrm{S}_{2}$ & 2.63 & 471.5 & 0.154 & HOMO-1 & $->$ & LUMO & $51.1 \%$ \\
\hline & & & & & HOMO & $->$ & LUMO & $26.5 \%$ \\
\hline & & & & & HOMO-2 & $->$ & LUMO & $23.1 \%$ \\
\hline & $\mathrm{S}_{3}$ & 3.22 & 384.6 & 0.203 & HOMO-2 & $->$ & LUMO & $64.3 \%$ \\
\hline & $\mathrm{S}_{9}$ & 3.86 & 321.4 & 0.090 & HOMO-4 & $->$ & LUMO & $41.4 \%$ \\
\hline & & & & & HOMO-8 & $->$ & LUMO & $12.0 \%$ \\
\hline & $\mathrm{S}_{15}$ & 4.73 & 262.2 & 0.092 & HOMO & $->$ & LUMO+6 & $61.6 \%$ \\
\hline & & & & & HOMO-10 & $->$ & LUMO & $14.4 \%$ \\
\hline \multirow[t]{12}{*}{ BLUE N2 } & $\mathrm{S}_{1}$ & 1.97 & 630.9 & 0.365 & HOMO & $->$ & LUMO & $55.5 \%$ \\
\hline & & & & & HOMO-3 & $->$ & LUMO & $12.2 \%$ \\
\hline & $\mathrm{S}_{5}$ & 3.25 & 381.9 & 0.327 & HOMO-4 & $->$ & LUMO & $59.5 \%$ \\
\hline & & & & & HOMO-3 & $->$ & LUMO & $21.1 \%$ \\
\hline & & & & & HOMO-8 & $->$ & LUMO & $19.0 \%$ \\
\hline & $\mathrm{S}_{9}$ & 3.50 & 354.7 & 0.104 & HOMO-9 & $->$ & LUMO & $55.2 \%$ \\
\hline & & & & & HOMO-6 & $->$ & LUMO & $16.5 \%$ \\
\hline & $\mathrm{S}_{13}$ & 4.09 & 303.2 & 0.151 & HOMO & $->$ & LUMO+2 & $39.6 \%$ \\
\hline & & & & & HOMO & $->$ & LUMO+4 & $27.7 \%$ \\
\hline & & & & & HOMO & $->$ & LUMO+5 & $25.0 \%$ \\
\hline & $\mathrm{S}_{15}$ & 4.37 & 283.6 & 0.279 & HOMO & $->$ & LUMO+5 & $57.2 \%$ \\
\hline & & & & & HOMO-10 & $->$ & LUMO & $12.8 \%$ \\
\hline
\end{tabular}




\begin{tabular}{|c|c|c|c|c|c|c|c|c|}
\hline \multicolumn{9}{|c|}{ ADF/PW91/ZORA/TZP } \\
\hline \multirow[b]{2}{*}{ BLUE N4 } & \multirow{2}{*}{\begin{tabular}{|l|} 
State \\
$\mathrm{S}_{1}$ \\
\end{tabular}} & \multirow{2}{*}{$\begin{array}{c}\begin{array}{c}\text { Energy } \\
(\mathrm{eV})\end{array} \\
1.6797\end{array}$} & \multirow{2}{*}{$\begin{array}{l}\begin{array}{c}\text { wavelength } \\
\text { (nm) }\end{array} \\
738 \\
\end{array}$} & \multirow{2}{*}{\begin{tabular}{|c|}
$\begin{array}{c}\text { Oscillator } \\
\text { Strength / } \\
\text { au }>\mathbf{0 . 0 8}\end{array}$ \\
0.16534 \\
\end{tabular}} & \multicolumn{4}{|c|}{$\begin{array}{c}\text { Transitions; main contributions }>10 \\
\%\end{array}$} \\
\hline & & & & & HOMO & $->$ & \begin{tabular}{|l|} 
LUMO \\
\end{tabular} & $89.27 \%$ \\
\hline & $\mathrm{S}_{3}$ & 2.5718 & 482 & 0.11387 & HOMO-2 & $->$ & LUMO & $50.31 \%$ \\
\hline & & & & & HOMO-4 & $->$ & LUMO & $27.65 \%$ \\
\hline & & & & & HOMO-3 & $->$ & LUMO & $13.32 \%$ \\
\hline & $\mathrm{S}_{5}$ & 2.6477 & 468 & 0.17463 & HOMO-3 & $->$ & LUMO & $36.80 \%$ \\
\hline & & & & & HOMO-2 & $->$ & LUMO & $30.10 \%$ \\
\hline & & & & & HOMO-4 & $\rightarrow$ & LUMO & $22.08 \%$ \\
\hline & $S_{10}$ & 3.3771 & 367 & 0.14054 & HOMO-8 & $->$ & LUMO & $28.18 \%$ \\
\hline & & & & & HOMO-7 & $->$ & LUMO & $19.17 \%$ \\
\hline & & & & & HOMO-6 & $->$ & LUMO & $18.72 \%$ \\
\hline & & & & & HOMO & $->$ & LUMO+1 & $10.24 \%$ \\
\hline & $\mathrm{S}_{15}$ & 3.8597 & 321 & 0.11408 & HOMO & $->$ & LUMO+6 & $49.79 \%$ \\
\hline & & & & & HOMO & $->$ & LUMO+4 & $23.74 \%$ \\
\hline & $\mathrm{S}_{16}$ & 3.8802 & 320 & \begin{tabular}{|l}
$9.04 \mathrm{E}-02$ \\
\end{tabular} & HOMO & $->$ & LUMO+6 & $47.88 \%$ \\
\hline & & & & & HOMO & $->$ & LUMO+4 & $15.95 \%$ \\
\hline & & & & & HOMO & $->$ & LUMO+7 & $10.72 \%$ \\
\hline & $\mathrm{S}_{18}$ & 4.0682 & 305 & 0.30031 & HOMO & $->$ & LUMO+7 & $51.46 \%$ \\
\hline & & & & & HOMO-11 & $->$ & LUMO & $17.56 \%$ \\
\hline & & & & & HOMO & $->$ & LUMO+4 & $13.09 \%$ \\
\hline \multirow[t]{13}{*}{ ORANGE } & $\mathrm{S}_{1}$ & 2.44 & 508 & 0.44565 & HOMO & $\rightarrow$ & LUMO & $74.51 \%$ \\
\hline & & & & & HOMO-1 & $->$ & LUMO & $13.57 \%$ \\
\hline & $\mathrm{S}_{3}$ & 2.93 & 423 & 0.32056 & HOMO-2 & $->$ & LUMO & $79.51 \%$ \\
\hline & $S_{16}$ & 4.20 & 295 & 0.17522 & HOMO & $\rightarrow$ & LUMO+6 & $43.92 \%$ \\
\hline & & & & & HOMO-1 & $->$ & LUMO+1 & $21.96 \%$ \\
\hline & & & & & HOMO & $\rightarrow$ & LUMO+7 & $11.75 \%$ \\
\hline & $\mathrm{S}_{19}$ & 4.34 & 286 & \begin{tabular}{|l|}
$8.06 \mathrm{E}-02$ \\
\end{tabular} & HOMO-1 & $->$ & LUMO+4 & $71.52 \%$ \\
\hline & & & & & HOMO-10 & $->$ & LUMO & $11.71 \%$ \\
\hline & & & & & HOMO & $->$ & LUMO+7 & $10.05 \%$ \\
\hline & $\mathrm{S}_{20}$ & 4.35 & 285 & \begin{tabular}{|l|}
$9.45 \mathrm{E}-02$ \\
\end{tabular} & HOMO-1 & $->$ & LUMO+3 & $36.13 \%$ \\
\hline & & & & & HOMO-10 & $->$ & LUMO & $17.43 \%$ \\
\hline & & & & & HOMO-1 & $->$ & LUMO+4 & $16.57 \%$ \\
\hline & & & & & HOMO & $->$ & LUMO+7 & $10.39 \%$ \\
\hline \multirow[t]{11}{*}{ BLUE N2 } & $\mathrm{S}_{1}$ & 1.8673 & 664 & 0.31388 & HOMO & $->$ & LUMO & $94.03 \%$ \\
\hline & $\mathrm{S}_{6}$ & 2.9455 & 421 & 0.1576 & HOMO-5 & $->$ & LUMO & $25.24 \%$ \\
\hline & & & & & HOMO-3 & $->$ & LUMO & $21.31 \%$ \\
\hline & & & & & HOMO-7 & $->$ & LUMO & $20.82 \%$ \\
\hline & & & & & HOMO-2 & $->$ & LUMO & $20.06 \%$ \\
\hline & $S_{16}$ & 3.929 & 316 & 0.46834 & HOMO & $->$ & LUMO+6 & $39.15 \%$ \\
\hline & & & & & HOMO & $->$ & LUMO+2 & $18.81 \%$ \\
\hline & & & & & HOMO-7 & $->$ & LUMO & $6.70 \%$ \\
\hline & & & & & HOMO-3 & $->$ & LUMO & $4.81 \%$ \\
\hline & & & & & HOMO & $->$ & LUMO+4 & $4.62 \%$ \\
\hline & & & & & & & & \\
\hline
\end{tabular}




\begin{tabular}{|c|c|c|c|c|c|c|c|c|}
\hline \multicolumn{9}{|c|}{ Gaussian/HF/SDD } \\
\hline \multirow[b]{2}{*}{ BLUE N4 } & \multirow{2}{*}{$\begin{array}{l}\text { State } \\
S_{2}\end{array}$} & \multirow{2}{*}{$\begin{array}{c}\begin{array}{c}\text { Energy } \\
(\mathbf{e V})\end{array} \\
3.91\end{array}$} & \multirow{2}{*}{$\begin{array}{l}\begin{array}{c}\text { wavelength } \\
\text { (nm) }\end{array} \\
317.16\end{array}$} & \multirow{2}{*}{\begin{tabular}{|c|}
$\begin{array}{c}\text { Oscillator } \\
\text { Strength / }\end{array}$ \\
au $>$ 0.08 \\
0.7987 \\
\end{tabular}} & \multicolumn{4}{|c|}{$\begin{array}{c}\text { Transitions; main contributions }>20 \\
\%\end{array}$} \\
\hline & & & & & HOMO & $->$ & LUMO & $62.7 \%$ \\
\hline & $\mathrm{S}_{3}$ & 5.32 & 232.86 & \begin{tabular}{|l|l|}
0.1614 \\
\end{tabular} & HOMO-6 & $->$ & LUMO & $59.9 \%$ \\
\hline & $\mathrm{S}_{8}$ & 5.91 & 209.93 & 0.1212 & HOMO & $->$ & LUMO+11 & $25.9 \%$ \\
\hline & & & & & HOMO-3 & $->$ & LUMO+9 & $20.6 \%$ \\
\hline & $\mathrm{S}_{15}$ & 6.79 & 182.61 & 0.1777 & HOMO & $->$ & LUMO+9 & $14.5 \%$ \\
\hline \multirow[t]{7}{*}{ ORANGE } & $\mathrm{S}_{2}$ & 4.29 & 289.06 & 1.2091 & HOMO & $->$ & LUMO & $60.5 \%$ \\
\hline & $\mathrm{S}_{4}$ & 5.55 & 223.56 & 0.2244 & HOMO-3 & $->$ & LUMO & $32.9 \%$ \\
\hline & & & & & HOMO-6 & $->$ & LUMO & $31.2 \%$ \\
\hline & $\mathrm{S}_{8}$ & 6.01 & 206.35 & 0.0909 & HOMO-2 & $->$ & LUMO+3 & $37.7 \%$ \\
\hline & & & & & HOMO-4 & $->$ & LUMO+5 & $32.5 \%$ \\
\hline & & & & & HOMO-4 & $->$ & $\mathrm{LUMO}+4$ & $30.2 \%$ \\
\hline & $\mathrm{S}_{14}$ & 6.73 & 184.14 & \begin{tabular}{|l|}
0.0851 \\
\end{tabular} & HOMO-2 & $->$ & LUMO+1 & $51.7 \%$ \\
\hline \multirow[t]{12}{*}{ BLUE N2 } & $\mathrm{S}_{2}$ & 4.21 & 294.51 & \begin{tabular}{|l|}
0.2519 \\
\end{tabular} & HOMO & $->$ & LUMO & $54.1 \%$ \\
\hline & & & & & HOMO-4 & $->$ & LUMO & $21.4 \%$ \\
\hline & $\mathrm{S}_{3}$ & 4.66 & 266.01 & 0.0976 & HOMO-3 & $->$ & LUMO & $46.1 \%$ \\
\hline & & & & & HOMO-1 & $->$ & LUMO & $27.3 \%$ \\
\hline & $\mathrm{S}_{4}$ & 4.94 & 250.96 & 0.7118 & HOMO-7 & $->$ & LUMO & $46.3 \%$ \\
\hline & $\mathrm{S}_{8}$ & 5.74 & 215.87 & 0.0841 & HOMO & $->$ & LUMO+1 & $44.6 \%$ \\
\hline & $\mathrm{S}_{9}$ & 5.95 & 208.43 & \begin{tabular}{|l|l|}
0.1419 \\
\end{tabular} & HOMO-4 & $->$ & LUMO & $27.6 \%$ \\
\hline & & & & & HOMO-7 & $->$ & LUMO & $25.7 \%$ \\
\hline & & & & & HOMO-2 & $->$ & LUMO+7 & $24.2 \%$ \\
\hline & & & & & HOMO-1 & $->$ & LUMO+2 & $20.2 \%$ \\
\hline & $\mathrm{S}_{14}$ & 6.82 & 181.92 & 0.4718 & HOMO-8 & $->$ & LUMO+3 & $43.4 \%$ \\
\hline & $\mathrm{S}_{15}$ & 6.84 & 181.14 & 0.1115 & HOMO-8 & $->$ & LUMO+3 & $15.6 \%$ \\
\hline
\end{tabular}

Table 6. Selected excitation energies (expressed as wavelength) for the strongest oscillator peaks (f) of the two blue forms of $\mathrm{PhHgHDz}, \mathrm{N} 4 \mathrm{H}$ and N2H.

\begin{tabular}{|c|c|c|c|c|c|c|c|c|}
\hline & \multicolumn{2}{|c|}{$\begin{array}{l}\text { ADF/PW91/ } \\
\text { ZORA/TZP }\end{array}$} & \multicolumn{2}{|c|}{$\begin{array}{l}\text { ADF/OLYP/ } \\
\text { ZORA/TZP }\end{array}$} & \multicolumn{2}{|c|}{$\begin{array}{c}\text { Gaussian/B3LYP/ } \\
\text { CEP-31G }\end{array}$} & \multicolumn{2}{|c|}{$\begin{array}{l}\text { Gaussian/ } \\
\text { HF/SSDD }\end{array}$} \\
\hline & $\lambda / \mathbf{n m}$ & f / a. u. & $\lambda / \mathbf{n m}$ & f / a. u. & $\lambda / \mathbf{n m}$ & f / a. u. & $\lambda / \mathrm{nm}$ & f / a. u. \\
\hline \multirow{6}{*}{ Blue N4H } & 738 & 0.165 & 709 & 0.182 & 623 & 0.298 & 317 & 0.799 \\
\hline & 482 & 0.114 & 471 & 0.207 & 432 & 0.291 & 233 & 0.161 \\
\hline & 468 & 0.175 & 457 & 0.092 & 334 & 0.184 & 210 & 0.121 \\
\hline & 367 & 0.141 & 365 & 0.102 & 288 & 0.082 & 183 & 0.178 \\
\hline & 321 & 0.114 & 322 & 0.107 & 283 & 0.083 & & \\
\hline & 305 & 0.300 & 303 & 0.286 & & & & \\
\hline \multirow{5}{*}{ Blue N2H } & 664 & 0.314 & 588 & 0.390 & 631 & 0.365 & 295 & 0.252 \\
\hline & 421 & 0.158 & 412 & 0.140 & 382 & 0.327 & 266 & 0.098 \\
\hline & 316 & 0.468 & 406 & 0.192 & 355 & 0.104 & 251 & 0.712 \\
\hline & & & 316 & 0.465 & 303 & 0.151 & 216 & 0.084 \\
\hline & & & 301 & 0.101 & 284 & 0.279 & 208 & 0.142 \\
\hline
\end{tabular}




\section{Optimized Cartesian coordinates}

1

$\mathrm{Hg}$

$\mathrm{S}$

$\mathrm{N}$

$\mathrm{N}$

$\mathrm{N}$

$\mathrm{C}$

C

C

C

C

C

C

$\mathrm{H}$

$\mathrm{H}$

$\mathrm{H}$

$\mathrm{H}$

$\mathrm{H}$

$\mathrm{H}$

$\mathrm{H}$

$\mathrm{H}$

$\mathrm{H}$

$\mathrm{H}$

$\mathrm{H}$

$\mathrm{H}$

$\mathrm{H}$

$\mathrm{Hg}$

S

$\mathrm{N}$

N

N

C

C

C

C

C

C

C

$\mathrm{H}$

$\mathrm{H}$

$\mathrm{H}$

$\mathrm{H}$

$\mathrm{H}$

$\mathrm{H}$

$\mathrm{H}$

$\mathrm{H}$

$\mathrm{H}$

$\mathrm{H}$

$\mathrm{H}$

H

$\mathrm{H}$

MeHg (cptsc) ADF (PW91)

0.551090000

$-0.501961000$

1.067709000

0.478009000

$-0.700648000$

$-0.183716000$

1.741818000

2.461049000

3.470902000

2.727762000

1.971621000

1.300540000

2. 909382000

1.739369000

4.368017000

2.009271000

2.577956000

3.792117000

3.397470000

1.028394000

$-0.753975000$

1.260146000

0.694378000

2.337084000

$-1.495158000$
5.659299000

4.913623000

7.545437000

7.401415000

6.111167000

6.291901000

8.631842000

8.962978000

10.044637000

10.819484000

9.727451000

5.913880000

8.081074000

9.386358000

9.567580000

11.513835000

9.319142000

10.679182000

11.412723000

10.056445000

6.961158000

6.976297000

5.336158000

5.563665000

5.485562000
4.950757000

2. 894851000

3.250005000

2. 016285000

0.551836000

1.816129000

3.416222000

4.702919000

4.281718000

3.178878000

2.399879000

6.915646000

5.174058000

5.422036000

3.860741000

3.638786000

1. 575171000

5.115918000

2. 545388000

1.945808000

$-0.002232000$

7.175388000

7.618810000

6.950227000

0.481524000

\section{MeHg (cptsc) Gaussian (ADF/B3LYP)}

0.556517000

$-0.682139000$

1.001455000

0.431688000

$-0.843335000$

$-0.286371000$

1.689383000

2.396011000

3.425700000

2.696075000

1.936940000

1.408988000

2.850689000

1.661748000

4.347007000

1.974931000

2.549587000

3.708422000

3.393115000

0.992327000

$-0.678480000$

1.203332000

0.962589000

2.497647000

$-1.401562000$
5.592124000

4.852105000

7.485131000

7.354142000

6.110879000

6.257141000

8.586879000

8.924209000

10.025954000

10.805653000

9.696326000

5.713870000

8.038130000

9.338817000

9.555540000

11.521700000

9.289230000

10.673780000

11.377860000

10.032052000

6.836186000

6.712827000

4.939899000

5.555088000

5.296372000
4.979424000

2.929140000

3. 310911000

2. 009274000

0.530973000

1.793466000

3.492413000

4.813612000

4.402881000

3.258419000

2.459015000

6.950199000

5.282005000

5.531176000

4.014461000

3.692444000

1.633826000

5.248518000

2.624972000

2.003462000

$-0.160710000$

7.367894000

7.593255000

6.878093000

0.305910000 


$\begin{array}{lr}\mathrm{Hg} & 0.561967000 \\ \mathrm{~S} & -0.633721000 \\ \mathrm{~N} & 1.025904000 \\ \mathrm{~N} & 0.447152000 \\ \mathrm{~N} & -0.810633000 \\ \mathrm{C} & -0.255588000 \\ \mathrm{C} & 1.698030000 \\ \mathrm{C} & 2.410283000 \\ \mathrm{C} & 3.404184000 \\ \mathrm{C} & 2.673533000 \\ \mathrm{C} & 1.930108000 \\ \mathrm{C} & 1.381292000 \\ \mathrm{H} & 2.881808000 \\ \mathrm{H} & 1.689654000 \\ \mathrm{H} & 4.320128000 \\ \mathrm{H} & 1.962674000 \\ \mathrm{H} & 2.532230000 \\ \mathrm{H} & 3.670049000 \\ \mathrm{H} & 3.350041000 \\ \mathrm{H} & 1.000074000 \\ \mathrm{H} & -0.652529000 \\ \mathrm{H} & 1.119660000 \\ \mathrm{H} & 0.986807000 \\ \mathrm{H} & 2.460684000 \\ \mathrm{H} & -1.361524000\end{array}$
5.607530000
4.913172000
7.495184000
7.355862000
6.166564000
6.298566000
8.564438000
8.899857000
10.009555000
10.767392000
9.664644000
5.727340000
8.035084000
9.281933000
9.568314000
11.468069000
9.271188000
10.648587000
11.334631000
9.990104000
6.880541000
6.681677000
4.927035000
5.639480000
5.375147000
4.988175000
2.919859000
3. 308100000
2. 038982000
0.570005000
1.809884000
3.482334000
4.779201000
4.377706000
3. 247220000
2.463332000
6.956135000
5. 222967000
5.496616000
3.998456000
3.673060000
1.651692000
5.209345000
2.621659000
2.020838000
$-0.102683000$
7.398744000
7.569463000
6.904182000
0.339762000

PhHg (cptsc) ADF (PW91)

$\begin{array}{lr}\mathrm{Hg} & 1.837331000 \\ \mathrm{~S} & 0.872727000 \\ \mathrm{~N} & 0.150116000 \\ \mathrm{~N} & 1.084235000 \\ \mathrm{~N} & 1.719882000 \\ \mathrm{C} & 0.716704000 \\ \mathrm{C} & 2.109797000 \\ \mathrm{C} & 2.828860000 \\ \mathrm{C} & 2.755905000 \\ \mathrm{C} & 2.796070000 \\ \mathrm{C} & 1.939142000 \\ \mathrm{C} & 2.597136000 \\ \mathrm{C} & 1.861117000 \\ \mathrm{C} & 2.349083000 \\ \mathrm{C} & 3.582713000 \\ \mathrm{C} & 4.327529000 \\ \mathrm{C} & 3.837566000 \\ \mathrm{H} & -0.180628000 \\ \mathrm{H} & -0.451508000 \\ \mathrm{H} & 2.408426000 \\ \mathrm{H} & 3.881160000 \\ \mathrm{H} & 3.561650000 \\ \mathrm{H} & 1.800872000 \\ \mathrm{H} & 2.438528000 \\ \mathrm{H} & 3.830889000 \\ \mathrm{H} & 2.205837000 \\ \mathrm{H} & 0.872318000 \\ \mathrm{H} & 0.897587000 \\ \mathrm{H} & 1.761747000 \\ \mathrm{H} & 3.962739000 \\ \mathrm{H} & 5.294248000 \\ \mathrm{H} & 4.438891000 \\ & \end{array}$

0.553826000

2.520027000

2.944621000

0.875776000

$-0.048566000$

1.986090000

$-1.122076000$

$-2.244968000$

$-3.414127000$

$-2.721209000$

$-1.457933000$

$-0.866055000$

$-1.216268000$

$-2.167558000$

$-2.779340000$

$-2.432789000$

$-1.484007000$

2.591650000

3.617466000

$-2.462010000$

$-1.952632000$

$-4.144709000$

$-3.945706000$

$-3.359909000$

$-2.431932000$

$-0.609811000$

$-1.662231000$

$-0.748298000$

$-2.428731000$

$-3.521391000$

$-2.902787000$

$-1.231170000$
1. 691129000

$-1.845610000$

$-1.613708000$

$-0.819397000$

$-1.032984000$

$-1.421887000$

$-0.713281000$

$-1.710781000$

$-3.083369000$

$-2.885063000$

3.040506000

4.182060000

5.081962000

4.856794000

3.728873000

2.827123000

$-2.738676000$

$-1.384602000$

0.275198000

$-0.557534000$

$-1.570098000$

$-1.589388000$

$-3.899908000$

$-3.320071000$

$-3.526722000$

$-3.074941000$

4.383039000

5.961797000

5.558093000

3. 547429000

1.953227000 


$\begin{array}{lrrr}\mathrm{Hg} & 1.828711000 & 0.592312000 & 1.704292000 \\ \mathrm{~S} & 0.785026000 & 2.635738000 & 0.719098000 \\ \mathrm{~N} & 0.132639000 & 3.079435000 & -1.878069000 \\ \mathrm{~N} & 1.098619000 & 0.989349000 & -1.632474000 \\ \mathrm{~N} & 1.674690000 & 0.010901000 & -0.767513000 \\ \mathrm{C} & 0.700794000 & 2.117038000 & -1.055963000 \\ \mathrm{C} & 2.064958000 & -1.086133000 & -1.372296000 \\ \mathrm{C} & 2.699871000 & -2.271746000 & -0.632061000 \\ \mathrm{C} & 2.555116000 & -3.452293000 & -1.646575000 \\ \mathrm{C} & 2.689140000 & -2.760053000 & -3.043917000 \\ \mathrm{C} & 1.943409000 & -1.396813000 & -2.871568000 \\ \mathrm{C} & 2.650777000 & -0.818942000 & 3.068325000 \\ \mathrm{C} & 1.800049000 & -1.618248000 & 3.896419000 \\ \mathrm{C} & 2.351464000 & -2.568693000 & 4.804015000 \\ \mathrm{C} & 3.761943000 & -2.730734000 & 4.900193000 \\ \mathrm{C} & 4.619171000 & -1.938881000 & 4.085658000 \\ \mathrm{C} & 4.067795000 & -0.990227000 & 3.176454000 \\ \mathrm{H} & 0.046160000 & 2.881733000 & -2.870595000 \\ \mathrm{H} & -0.187876000 & 3.960644000 & -1.495420000 \\ \mathrm{H} & 2.229521000 & -2.457279000 & 0.344804000 \\ \mathrm{H} & 3.770278000 & -2.061587000 & -0.443653000 \\ \mathrm{H} & 3.309884000 & -4.238744000 & -1.484742000 \\ \mathrm{H} & 1.557497000 & -3.916719000 & -1.546245000 \\ \mathrm{H} & 2.268543000 & -3.368575000 & -3.860872000 \\ \mathrm{H} & 3.755203000 & -2.582952000 & -3.274831000 \\ \mathrm{H} & 2.350903000 & -0.575482000 & -3.481359000 \\ \mathrm{H} & 0.872937000 & -1.475089000 & -3.136272000 \\ \mathrm{H} & 0.713817000 & -1.505881000 & 3.844097000 \\ \mathrm{H} & 1.685590000 & -3.170145000 & 5.429116000 \\ \mathrm{H} & 4.185122000 & -3.457357000 & 5.599172000 \\ \mathrm{H} & 5.704400000 & -2.053659000 & 4.157126000 \\ \mathrm{H} & 4.745850000 & -0.389917000 & 2.563658000\end{array}$

\section{6}

$\mathrm{Hg}$

$\mathrm{S}$

$\mathrm{N}$

$\mathrm{N}$

$\mathrm{N}$

C

C

C

C

C

C

C

C

C

C

C

$\mathrm{H}$
$\mathrm{H}$

$\mathrm{H}$

$\mathrm{H}$

$\mathrm{H}$

$\mathrm{H}$

$\mathrm{H}$

$\mathrm{H}$

$\mathrm{H}$

\section{PhHg (cptsc) Gaussian (HF/SSD)}

1.833002000

0.804497000

0.202021000

1.130078000

1.680106000

0.741575000

2.048953000

2.659913000

2. 522001000

2.657169000

1.935687000

2.657623000

1.833778000

2.377793000

3.761023000

4.595214000

4.048287000

0.127046000

$-0.110940000$

2.186299000

3. 708110000

3.260653000

1. 542848000

2. 238087000

3. 705382000

2.350066000
0.587358000

2. 579897000

3.023262000

0.968727000

$-0.012329000$

2.073362000

$-1.082033000$

$-2.267411000$

$-3.424836000$

$-2.733247000$

$-1.380251000$

$-0.820121000$

$-1.666147000$

$-2.602308000$

$-2.711028000$

$-1.876972000$

$-0.942762000$

2. 829488000

3.891452000

$-2.460282000$

$-2.064771000$

$-4.199709000$

$-3.883595000$

$-3.321430000$

$-2.570995000$

$-0.578099000$
1.720946000

0.682992000

$-1.879203000$

$-1.598603000$

$-0.760650000$

$-1.060890000$

$-1.350088000$

$-0.627542000$

$-1.637968000$

$-3.012475000$

$-2.833773000$

3.067310000

3.828698000

4.711207000

4.853899000

4.109246000

3. 226799000

$-2.850891000$

$-1.516930000$

0.323272000

$-0.428425000$

$-1.482025000$

$-1.546451000$

$-3.818193000$

$-3.241686000$

$-3.426657000$ 
4.214790000

2.670473000

\section{PhHg (ptsc) ADF (PW91)}

$\begin{array}{lr}\mathrm{Hg} & 1.368964000 \\ \mathrm{~S} & -0.692493000 \\ \mathrm{~N} & -1.196197000 \\ \mathrm{~N} & 0.983610000 \\ \mathrm{~N} & 1.964915000 \\ \mathrm{C} & 4.828420000 \\ \mathrm{C} & -0.180137000 \\ \mathrm{C} & 3.128449000 \\ \mathrm{C} & 3.568144000 \\ \mathrm{~N} & 2.773293000 \\ \mathrm{C} & 3.481654000 \\ \mathrm{C} & 4.768905000 \\ \mathrm{C} & 2.912343000 \\ \mathrm{C} & 4.228997000 \\ \mathrm{C} & 5.267126000 \\ \mathrm{C} & 5.002022000 \\ \mathrm{C} & 3.696018000 \\ \mathrm{C} & 2.657802000 \\ \mathrm{H} & -0.903206000 \\ \mathrm{H} & -1.966616000 \\ \mathrm{H} & 5.681413000 \\ \mathrm{H} & 3.872812000 \\ \mathrm{H} & 1.806161000 \\ \mathrm{H} & 3.034927000 \\ \mathrm{H} & 5.566716000 \\ \mathrm{H} & 4.463054000 \\ \mathrm{H} & 6.284727000 \\ \mathrm{H} & 5.810990000 \\ \mathrm{H} & 3.481024000 \\ \mathrm{H} & 1.645590000\end{array}$

0.818054000

$-0.421064000$

$-2.483160000$

$-1.816211000$

$-0.914415000$

$-2.186797000$

$-1.635331000$

$-1.063948000$

$-2.042712000$

$-3.062751000$

$-3.832815000$

$-3.310486000$

2.099293000

1.870465000

2.714033000

3.797402000

4.033072000

3.190715000

$-3.340410000$

$-2.574152000$

$-1.539110000$

$-0.321156000$

$-3.132032000$

$-4.690134000$

$-3.707252000$

1.026851000

2.521971000

4.454519000

4.875497000

3.393916000
1.527489000

1.854462000

3. 412182000

3. 617849000

3. 295529000

5.415148000

3.036514000

3. 874568000

4.815129000

5.296448000

6.166266000

6.261002000

0.921196000

1.345128000

0.941701000

0.102969000

$-0.327693000$

0.078654000

3.871523000

2.759878000

5. 245291000

3. 590113000

4.978497000

6.654054000

6.877222000

1.994105000

1.282122000

$-0.214637000$

$-0.984469000$

$-0.270085000$

\section{8}

$\mathrm{Hg}$

$\mathrm{S}$

$\mathrm{N}$

$\mathrm{N}$

$\mathrm{N}$

C

C

C

C

$\mathrm{N}$

C

C

C

C

C

C

C

C

$\mathrm{H}$

$\mathrm{H}$

\section{PhHg (ptsc) Gaussian (ADF/B3LYP)}

1.405197000

$-0.728294000$

$-1.204803000$

0.947408000

1.938830000

4.751677000

$-0.215196000$

3.093154000

3.506354000

2.699176000

3.385045000

4.672214000

2.924481000

4.280358000

5.314083000

5.007341000

3.663171000

2.627953000

$-1.019197000$

$-2.104951000$
0.683622000

$-0.619100000$

$-2.748156000$

$-1.939209000$

$-0.984386000$

$-2.012356000$

$-1.831837000$

$-1.046225000$

- 1.961674000

$-2.987883000$

$-3.675289000$

$-3.091865000$

2.046830000

1.600304000

2.503486000

3.865945000

4.321550000

3.418640000

$-3.460741000$

$-2.709498000$
1.386572000

1. 599084000

3.205018000

3.533684000

3.172540000

5.575258000

2. 886116000

3.827900000

4.881017000

5.398184000

6.382923000

6.519862000

0.799780000

0.689075000

0.307013000

0.034372000

0.140892000

0.518963000

3.904191000

2. 741652000 


$\begin{array}{lrrr}\mathrm{H} & 5.594454000 & -1.346892000 & 5.407730000 \\ \mathrm{H} & 3.828323000 & -0.294405000 & 3.529699000 \\ \mathrm{H} & 1.754572000 & -3.118029000 & 5.026718000 \\ \mathrm{H} & 2.936293000 & -4.510456000 & 6.913227000 \\ \mathrm{H} & 5.443384000 & -3.411908000 & 7.214210000 \\ \mathrm{H} & 4.536131000 & 0.555359000 & 0.884779000 \\ \mathrm{H} & 6.343728000 & 2.144942000 & 0.222617000 \\ \mathrm{H} & 5.800476000 & 4.559311000 & -0.258284000 \\ \mathrm{H} & 3.419784000 & 5.366677000 & -0.069448000 \\ \mathrm{H} & 1.601852000 & 3.787244000 & 0.590654000\end{array}$

\section{9}

$\mathrm{Hg}$

$\mathrm{S}$

$\mathrm{N}$

$\mathrm{N}$

$\mathrm{N}$

C

C

C

C

$\mathrm{N}$

C

C

C

C

C

C

C

C

$\mathrm{H}$

$\mathrm{H}$

$\mathrm{H}$

$\mathrm{H}$

$\mathrm{H}$

$\mathrm{H}$

$\mathrm{H}$

$\mathrm{H}$

$\mathrm{H}$

$\mathrm{H}$

$\mathrm{H}$

$\mathrm{H}$

10

$\begin{array}{ll}\mathrm{Hg} & 1.175283000 \\ \mathrm{~S} & 1.220787000 \\ \mathrm{~N} & 3.706066000 \\ \mathrm{~N} & 3.983717000 \\ \mathrm{C} & 2.954649000 \\ \mathrm{~N} & 3.389886000 \\ \mathrm{~N} & 2.490208000 \\ \mathrm{H} & 1.502403000 \\ \mathrm{C} & 0.653133000 \\ \mathrm{C} & 4.813583000 \\ \mathrm{C} & 4.600217000 \\ \mathrm{C} & 5.644840000 \\ \mathrm{C} & 6.907922000 \\ \mathrm{C} & 7.120081000 \\ \mathrm{C} & 6.086456000 \\ \mathrm{C} & 2.831043000\end{array}$

MeHgHDz ADF (PW91)
0.670598000

$-0.656385000$

$-2.752409000$

$-1.937801000$

$-1.003371000$

$-1.935087000$

$-1.848966000$

$-1.047384000$

$-1.940689000$

$-2.967355000$

$-3.602390000$

$-2.989253000$

2.020217000

1.562847000

2.445483000

3.807607000

4.279929000

3.395047000

$-3.448429000$

$-2.724157000$

$-1.256467000$

$-0.309056000$

$-3.157721000$

$-4.429897000$

$-3.261377000$

0.519183000

2.071747000

4.486827000

5.325961000

3.786748000
1.353879000

1.591302000

3.165088000

3.496447000

3. 170112000

5.544038000

2.863271000

3.819562000

4.876262000

5.400927000

6.376424000

6.496197000

0.802936000

0.576922000

0.220433000

0.083913000

0.303300000

0.657125000

3.851683000

2.704926000

5.368240000

3.540346000

5.067493000

6.914402000

7.176815000

0.669472000

0.050075000

$-0.189733000$

0.198506000

0.815636000
1.865385000
1.874780000
0.069026000
3.510727000
1.278337000
2.193482000
2.941126000
3. 599106000
$-0.337576000$
4.367692000
5.061436000
4.971892000
0.507143000
1. 532243000
0.886035000
0.217097000
0.172948000
0.806224000
1.485665000
5.915541000 
7.469939000

3.421395000

$-5.153445000$

7.649011000

4.444347000

$-4.500209000$

6.955011000

4.162376000

$-3.448087000$

6.089165000

1.099063000

3.157139000

3.443994000

1.039156000

4.348737000

3.532282000

3.610289000

4.670326000

3.608618000

1. 419241000

0.299615000

8.102697000

$-2.930830000$

4.947799000

$-4.814744000$

5.478665000

$-5.975449000$

3.653950000

$-5.244145000$

1.292171000

$-3.372226000$

$-0.464278000$

0.416048000

0.874018000

0.927830000

$-0.271354000$

$-0.355317000$

0.764948000

1.983614000

5.543975000

7.089569000

8.323758000

8.004019000

6.466405000

\section{MeHgHDz Gaussian (ADF/B3LYP)}

$\begin{array}{lr}\mathrm{Hg} & 1.113336000 \\ \mathrm{~S} & 1.025454000 \\ \mathrm{~N} & 3.589833000 \\ \mathrm{~N} & 3.868072000 \\ \mathrm{C} & 2.818445000 \\ \mathrm{~N} & 3.255866000 \\ \mathrm{~N} & 2.351688000 \\ \mathrm{H} & 1.357038000 \\ \mathrm{C} & 0.589533000 \\ \mathrm{C} & 4.735075000 \\ \mathrm{C} & 4.481321000 \\ \mathrm{C} & 5.555911000 \\ \mathrm{C} & 6.886391000 \\ \mathrm{C} & 7.137124000 \\ \mathrm{C} & 6.072686000 \\ \mathrm{C} & 2.764094000 \\ \mathrm{C} & 1.762159000 \\ \mathrm{C} & 2.140266000 \\ \mathrm{C} & 3.506164000 \\ \mathrm{C} & 4.494736000 \\ \mathrm{C} & 4.137648000 \\ \mathrm{H} & 0.896236000 \\ \mathrm{H} & -0.498768000 \\ \mathrm{H} & 1.112261000 \\ \mathrm{H} & 3.458764000 \\ \mathrm{H} & 5.356536000 \\ \mathrm{H} & 7.713928000 \\ \mathrm{H} & 8.158028000 \\ \mathrm{H} & 6.248637000 \\ \mathrm{H} & 4.884549000 \\ \mathrm{H} & 5.545340000 \\ \mathrm{H} & 3.793803000 \\ \mathrm{H} & 1.372499000 \\ \mathrm{H} & 0.712352000\end{array}$

2.056707000

0.138553000

1.297960000

0.294386000

$-0.326499000$

$-1.335010000$

$-2.031681000$

$-1.780440000$

3.698704000

1.873408000

2. 973746000

3.593697000

3.120054000

2. 020075000

1. 392932000

$-3.111527000$

$-3.808205000$

$-4.889228000$

$-5.278990000$

$-4.574339000$

$-3.490745000$

3.456674000

3.857060000

4.604028000

3.330010000

4.435079000

3.596694000

1. 655024000

0.552719000

$-2.945561000$

$-4.867223000$

$-6.111224000$

$-5.421141000$

$-3.510667000$
1.917320000

3. 531321000

2. 149183000

2.940029000

3.627537000

4.396554000

5.123527000

5.075562000

0.633272000

1.484005000

0.609555000

$-0.081188000$

0.096521000

0.971153000

1.666253000

5.956013000

6.698787000

7.537838000

7.640926000

6.893683000

6.049077000

$-0.397179000$

0.671988000

0.981997000

0.479496000

$-0.748943000$

$-0.434788000$

1.108858000

2.336881000

5.473855000

6.967225000

8.287434000

8.105398000

6.621249000

\section{2}

\section{MeHgHDz Gaussian (HF/SSD)}

$\mathrm{Hg}$

$\mathrm{S}$

$\mathrm{N}$
0.949735000

0.957414000

3.556422000
1.992885000

0.110225000

1. 218657000
1.870808000

3.466270000

2.178823000 


$\begin{array}{lr}\mathrm{N} & 3.777130000 \\ \mathrm{C} & 2.711201000 \\ \mathrm{~N} & 3.109555000 \\ \mathrm{~N} & 2.267271000 \\ \mathrm{H} & 1.288271000 \\ \mathrm{C} & 0.530266000 \\ \mathrm{C} & 4.705689000 \\ \mathrm{C} & 4.475471000 \\ \mathrm{C} & 5.544042000 \\ \mathrm{C} & 6.843304000 \\ \mathrm{C} & 7.069965000 \\ \mathrm{C} & 6.008022000 \\ \mathrm{C} & 2.748797000 \\ \mathrm{C} & 1.827182000 \\ \mathrm{C} & 2.265971000 \\ \mathrm{C} & 3.623264000 \\ \mathrm{C} & 4.535853000 \\ \mathrm{C} & 4.111095000 \\ \mathrm{H} & 0.865106000 \\ \mathrm{H} & -0.535848000 \\ \mathrm{H} & 1.044950000 \\ \mathrm{H} & 3.476137000 \\ \mathrm{H} & 5.362042000 \\ \mathrm{H} & 7.666869000 \\ \mathrm{H} & 8.068591000 \\ \mathrm{H} & 6.172415000 \\ \mathrm{H} & 4.811479000 \\ \mathrm{H} & 5.582544000 \\ \mathrm{H} & 3.960109000 \\ \mathrm{H} & 1.549816000 \\ \mathrm{H} & 0.780400000\end{array}$

13

PhHgHDz (orange)

$\begin{array}{lr}\mathrm{Hg} & 1.148412000 \\ \mathrm{~S} & 2.181361000 \\ \mathrm{~N} & -0.064240000 \\ \mathrm{~N} & 0.442339000 \\ \mathrm{C} & 1.501380000 \\ \mathrm{~N} & 2.013455000 \\ \mathrm{~N} & 3.075091000 \\ \mathrm{C} & 0.610844000 \\ \mathrm{C} & -0.716162000 \\ \mathrm{C} & -1.055947000 \\ \mathrm{C} & -0.075860000 \\ \mathrm{C} & 1.247541000 \\ \mathrm{C} & 1.589928000 \\ \mathrm{C} & -1.156022000 \\ \mathrm{C} & -1.609819000 \\ \mathrm{C} & -2.680891000 \\ \mathrm{C} & -3.316017000 \\ \mathrm{C} & -2.868755000 \\ \mathrm{C} & -1.796199000 \\ \mathrm{C} & 3.761872000 \\ \mathrm{C} & 4.907781000 \\ \mathrm{C} & 5.627356000 \\ \mathrm{C} & 5.213899000 \\ \mathrm{C} & 4.067967000 \\ \mathrm{C} & 3.336379000 \\ \mathrm{H} & 3.474045000 \\ \mathrm{H} & -1.499790000 \\ \mathrm{H} & -2.091531000\end{array}$

0.272719000

$-0.357396000$

$-1.309982000$

$-2.040558000$

$-1.850751000$

3.611856000

1.794295000

2.857069000

3.464457000

3.007926000

1.940646000

1.331233000

$-3.078101000$

$-3.816231000$

$-4.852384000$

$-5.163981000$

$-4.421780000$

$-3.382582000$

3.361537000

3.798791000

4.501191000

3.204947000

4.281998000

3.473089000

1.587783000

0.514529000

$-2.815412000$

$-4.650375000$

$-5.961791000$

$-5.411581000$

$-3.584057000$
2.951387000

3.628236000

4.382682000

5.114755000

5.084692000

0.553946000

1.537005000

0.666744000

0.008805000

0.222015000

1.096567000

1.755346000

5.930125000

6.679198000

7.499106000

7.581862000

6.830549000

6.006184000

$-0.445767000$

0.538093000

0.898017000

0.505514000

$-0.659985000$

$-0.282565000$

1. 262114000

2.425783000

5.431645000

6.884452000

8.213303000

8.068992000

6.622270000

\section{ADF (PW91)}

$\begin{array}{rr}12.102379000 & 0.060186000 \\ 10.115194000 & 0.990505000 \\ 11.693233000 & 2.342461000 \\ 10.807786000 & 3.109033000 \\ 10.076666000 & 2.640596000 \\ 9.295263000 & 3.580594000 \\ 8.580764000 & 3.265823000 \\ 13.747177000 & -1.128674000 \\ 13.952819000 & -1.532702000 \\ 15.061032000 & -2.314179000 \\ 15.976184000 & -2.700139000 \\ 15.777726000 & -2.304744000 \\ 14.668579000 & -1.525536000 \\ 12.402171000 & 2.889484000 \\ 13.520092000 & 2.174034000 \\ 14.269963000 & 2.651383000 \\ 13.906338000 & 3.840270000 \\ 12.786511000 & 4.552833000 \\ 12.036042000 & 4.088529000 \\ 7.815892000 & 4.212010000 \\ 7.120669000 & 3.797714000 \\ 6.365819000 & 4.718467000 \\ 6.294907000 & 6.050662000 \\ 6.986128000 & 6.453509000 \\ 7.746765000 & 5.546269000 \\ 8.685022000 & 2.324221000 \\ 13.251616000 & -1.244409000 \\ 15.206501000 & -2.622533000\end{array}$




$\begin{array}{lr}\mathrm{H} & -0.341318000 \\ \mathrm{H} & 2.020296000 \\ \mathrm{H} & 2.630647000 \\ \mathrm{H} & -1.102607000 \\ \mathrm{H} & -3.017854000 \\ \mathrm{H} & -4.157273000 \\ \mathrm{H} & -3.365573000 \\ \mathrm{H} & -1.436059000 \\ \mathrm{H} & 5.230917000 \\ \mathrm{H} & 6.518329000 \\ \mathrm{H} & 5.781499000 \\ \mathrm{H} & 3.740420000 \\ \mathrm{H} & 2.447596000\end{array}$

14

PhHgHDz (orange)
16.840173000

16.485779000

14.531776000

13.803518000

15.142883000

14.489732000

12.498831000

11.163911000

7.179081000

5.831815000

5.708103000

6.937694000

8.296447000
$-3.309112000$

$-2.603598000$

$-1.231783000$

1.252687000

2.093362000

4.212534000

5.478806000

4.629662000

2.757336000

4.391949000

6.770238000

7.491360000

5.846745000
$\mathrm{Hg}$

$\mathrm{S}$

$\mathrm{N}$

$\mathrm{N}$

C

$\mathrm{N}$

$\mathrm{N}$

C

C

C

C

C

C

C

C

C

C

C

C

C

C

C

C

$\mathrm{H}$

$\mathrm{H}$

$\mathrm{H}$

$\mathrm{H}$

$\mathrm{H}$

$\mathrm{H}$

$\mathrm{H}$

$\mathrm{H}$

$\mathrm{H}$

$\mathrm{H}$

$\mathrm{H}$

$\mathrm{H}$

$\mathrm{H}$

$\mathrm{H}$

$\mathrm{H}$

$\mathrm{H}$
$-1.311571000$

1.132524000

$-0.181577000$

1.123984000

1.851530000

3.174037000

4.007857000

$-3.428224000$

$-4.176479000$

$-5.594878000$

$-6.282683000$

$-5.547916000$

$-4.128970000$

$-0.844868000$

$-2.270104000$

$-2.989991000$

$-2.295052000$

$-0.870496000$

$-0.139626000$

5.420945000

6.247964000

7.659944000

8.250898000

7.412666000

5.998765000

3.621093000

$-3.668271000$

$-6.152081000$

$-7.371041000$

$-6.069256000$

$-3.583311000$

$-2.798861000$

$-4.079694000$

$-2.849655000$

$-0.334814000$

0.947483000

5.796895000

8.291340000

9.337729000

7.858093000

5.348996000

\section{Gaussian (ADF/B3LYP)}

$-1.019865000$

$-1.533617000$

1.311752000

1.373785000

0.178052000

0.397193000

$-0.668668000$

$-1.203255000$

$-0.666447000$

$-0.796414000$

$-1.460596000$

$-1.998247000$

$-1.873696000$

2.594676000

2.579104000

3.803051000

5.043270000

5.054524000

3.840399000

$-0.486877000$

$-1.651500000$

$-1.504671000$

$-0.208922000$

0.943913000

0.818867000

$-1.619873000$

$-0.155262000$

$-0.384409000$

$-1.559441000$

$-2.511607000$

$-2.299041000$

1.627070000

3.785575000

5.984761000

6.005723000

3.833308000

$-2.648034000$

$-2.396624000$

$-0.099632000$

1.942138000

1.692621000
0.001979000

$-0.027066000$

$-0.061608000$

$-0.043452000$

$-0.024784000$

$-0.008358000$

0.009520000

0.053438000

1. 148908000

1. 189475000

0.135434000

$-0.958259000$

$-0.998508000$

$-0.083364000$

$-0.175561000$

$-0.202914000$

$-0.134982000$

$-0.041569000$

$-0.016021000$

0.025101000

0.046895000

0.062166000

0.055940000

0.034142000

0.018537000

0.013135000

1.970703000

2.035237000

0.167060000

$-1.770724000$

$-1.844380000$

$-0.233969000$

$-0.276989000$

$-0.154906000$

0.010879000

0.052905000

0.051829000

0.078780000

0.067605000

0.028989000

0.001460000 


$\begin{array}{lrrr}\mathrm{Hg} & 1.336951000 & -1.111824000 & -0.000134000 \\ \mathrm{~S} & -1.074292000 & -1.548196000 & -0.000728000 \\ \mathrm{~N} & 0.101358000 & 1.326461000 & -0.000456000 \\ \mathrm{~N} & -1.139525000 & 1.323511000 & -0.000349000 \\ \mathrm{C} & -1.848642000 & 0.103455000 & -0.000367000 \\ \mathrm{~N} & -3.115446000 & 0.272979000 & -0.000131000 \\ \mathrm{~N} & -3.975294000 & -0.746661000 & -0.000070000 \\ \mathrm{C} & 3.443609000 & -1.133016000 & 0.000367000 \\ \mathrm{C} & 4.166671000 & -1.142070000 & -1.203879000 \\ \mathrm{C} & 5.563112000 & -1.154017000 & -1.204446000 \\ \mathrm{C} & 6.264818000 & -1.158163000 & 0.001122000 \\ \mathrm{C} & 5.562499000 & -1.150724000 & 1.206319000 \\ \mathrm{C} & 4.166058000 & -1.138757000 & 1.205004000 \\ \mathrm{C} & 0.734216000 & 2.616637000 & -0.000411000 \\ \mathrm{C} & 2.126951000 & 2.636412000 & -0.000916000 \\ \mathrm{C} & 2.805146000 & 3.854788000 & -0.000905000 \\ \mathrm{C} & 2.090335000 & 5.050839000 & -0.000381000 \\ \mathrm{C} & 0.691968000 & 5.025424000 & 0.000132000 \\ \mathrm{C} & 0.009826000 & 3.814230000 & 0.000120000 \\ \mathrm{C} & -5.360245000 & -0.509361000 & 0.000170000 \\ \mathrm{C} & -6.222331000 & -1.610187000 & 0.000386000 \\ \mathrm{C} & -7.600949000 & -1.415591000 & 0.000600000 \\ \mathrm{C} & -8.134307000 & -0.126541000 & 0.000625000 \\ \mathrm{C} & -7.265923000 & 0.966167000 & 0.000408000 \\ \mathrm{C} & -5.884746000 & 0.786374000 & 0.000177000 \\ \mathrm{H} & -3.636537000 & -1.684978000 & -0.000225000 \\ \mathrm{H} & 3.654148000 & -1.141499000 & -2.146809000 \\ \mathrm{H} & 6.094764000 & -1.161903000 & -2.136298000 \\ \mathrm{H} & 7.337329000 & -1.168975000 & 0.001409000 \\ \mathrm{H} & 6.093675000 & -1.156061000 & 2.138460000 \\ \mathrm{H} & 3.653060000 & -1.135596000 & 2.147670000 \\ \mathrm{H} & 2.675590000 & 1.717530000 & -0.001322000 \\ \mathrm{H} & 3.876767000 & 3.865016000 & -0.001304000 \\ \mathrm{H} & 2.610140000 & 5.988497000 & -0.000369000 \\ \mathrm{H} & 0.140113000 & 5.944501000 & 0.000539000 \\ \mathrm{H} & -1.059153000 & 3.785244000 & 0.000504000 \\ \mathrm{H} & -5.821478000 & -2.606300000 & 0.000387000 \\ \mathrm{H} & -8.252508000 & -2.267518000 & 0.000763000 \\ \mathrm{H} & -9.195676000 & 0.022294000 & 0.000800000 \\ \mathrm{H} & -7.662226000 & 1.962973000 & 0.000416000 \\ \mathrm{H} & -5.221448000 & 1.624438000 & 0.000010000\end{array}$

16 PhHgHDz (blue-N (4) H) ADF (PW91)

$\begin{array}{lr}\mathrm{Hg} & 1.160469000 \\ \mathrm{~S} & 2.087606000 \\ \mathrm{~N} & -0.163321000 \\ \mathrm{~N} & 0.302291000 \\ \mathrm{C} & 1.335549000 \\ \mathrm{~N} & 1.853006000 \\ \mathrm{~N} & 1.353239000 \\ \mathrm{C} & 0.641086000 \\ \mathrm{C} & -0.685186000 \\ \mathrm{C} & -1.010788000 \\ \mathrm{C} & -0.016338000 \\ \mathrm{C} & 1.306689000 \\ \mathrm{C} & 1.633137000 \\ \mathrm{C} & -1.256005000 \\ \mathrm{C} & -1.604800000 \\ \mathrm{C} & -2.670145000 \\ \mathrm{C} & -3.409035000\end{array}$

12.056722000

10.107097000

11.832830000

10.933495000

10.149356000

9.287830000

9.280248000

13.710225000

13.946813000

15.067180000

15.964992000

15.736211000

14.616312000

12.562358000

13.730293000

14.514061000

14.137236000
0.054885000

1. 110597000

2.363909000

3.151079000

2.723808000

3.598341000

4.818836000

$-1.130239000$

$-1.522167000$

$-2.293040000$

$-2.682858000$

$-2.302296000$

$-1.533031000$

2. 863134000

2.162833000

2. 594569000

3. 717964000 


$\begin{array}{lr}\mathrm{C} & -3.076759000 \\ \mathrm{C} & -2.011108000 \\ \mathrm{C} & 1.816677000 \\ \mathrm{C} & 1.195457000 \\ \mathrm{C} & 1.630764000 \\ \mathrm{C} & 2.683624000 \\ \mathrm{C} & 3.295723000 \\ \mathrm{C} & 2.872089000 \\ \mathrm{H} & 0.599433000 \\ \mathrm{H} & -1.480216000 \\ \mathrm{H} & -2.046074000 \\ \mathrm{H} & -0.270022000 \\ \mathrm{H} & 2.090695000 \\ \mathrm{H} & 2.673756000 \\ \mathrm{H} & -1.018855000 \\ \mathrm{H} & -2.923968000 \\ \mathrm{H} & -4.246639000 \\ \mathrm{H} & -3.662738000 \\ \mathrm{H} & -1.759007000 \\ \mathrm{H} & 0.374987000 \\ \mathrm{H} & 1.144727000 \\ \mathrm{H} & 3.025204000 \\ \mathrm{H} & 4.119002000 \\ \mathrm{H} & 3.342365000\end{array}$

$\begin{array}{rr}12.964494000 & 4.408176000 \\ 12.178893000 & 3.989404000 \\ 8.448217000 & 5.833293000 \\ 8.546225000 & 7.090507000 \\ 7.748712000 & 8.142300000 \\ 6.849858000 & 7.955530000 \\ 6.757172000 & 6.700975000 \\ 7.546270000 & 5.636198000 \\ 9.945532000 & 5.019379000 \\ 13.258983000 & -1.232363000 \\ 15.235693000 & -2.591427000 \\ 16.838342000 & -3.282636000 \\ 16.430117000 & -2.604762000 \\ 14.457480000 & -1.249761000 \\ 14.017659000 & 1.290922000 \\ 15.423134000 & 2.051319000 \\ 14.747772000 & 4.052925000 \\ 12.660706000 & 5.275360000 \\ 11.255951000 & 4.506865000 \\ 9.251297000 & 7.233609000 \\ 7.831635000 & 9.113310000 \\ 6.227854000 & 8.780439000 \\ 6.059763000 & 6.550026000 \\ 7.485606000 & 4.657765000\end{array}$

\section{PhHgHDz (blue-N (4)H) Gaussian (ADF/B3LYP)}

\begin{tabular}{|c|c|}
\hline $\mathrm{Hg}$ & 1.560674000 \\
\hline $\mathrm{S}$ & -0.700656000 \\
\hline $\mathrm{N}$ & -0.067096000 \\
\hline $\mathrm{N}$ & -1.350280000 \\
\hline $\mathrm{C}$ & -1.768497000 \\
\hline $\mathrm{N}$ & -3.08937400 \\
\hline $\mathrm{N}$ & -3.96135500 \\
\hline C & 3.6396240 \\
\hline $\mathrm{C}$ & 4.33893400 \\
\hline $\mathrm{C}$ & 5.73257400 \\
\hline $\mathrm{C}$ & 6.44681400 \\
\hline $\mathrm{C}$ & 5.76353300 \\
\hline $\mathrm{C}$ & 4.37011700 \\
\hline $\mathrm{C}$ & 0.2807510 \\
\hline C & 1.68027700 \\
\hline $\mathrm{C}$ & 2.11895700 \\
\hline $\mathrm{C}$ & 1.16965300 \\
\hline $\mathrm{C}$ & -0.22718600 \\
\hline $\mathrm{C}$ & -0.67739300 \\
\hline $\mathrm{C}$ & -5.36525100 \\
\hline $\mathrm{C}$ & -6.2283650 \\
\hline C & -7.63540300 \\
\hline $\mathrm{C}$ & -8.18705700 \\
\hline $\mathrm{C}$ & -7.31373300 \\
\hline $\mathrm{C}$ & -5.90460500 \\
\hline $\mathrm{H}$ & -3.59042800 \\
\hline $\mathrm{H}$ & 3.8112250 \\
\hline $\mathrm{H}$ & 6.2512520 \\
\hline $\mathrm{H}$ & 7.51647700 \\
\hline $\mathrm{H}$ & 6.30590100 \\
\hline $\mathrm{H}$ & 3.86620400 \\
\hline $\mathrm{H}$ & 2.4008270 \\
\hline $\mathrm{H}$ & 3.18889800 \\
\hline $\mathrm{H}$ & 1.50793400 \\
\hline $\mathrm{H}$ & -0.956772 \\
\hline
\end{tabular}

$-0.019432000$

$-0.051009000$

$-0.008200000$

$-0.007685000$

$-0.021718000$

$-0.015630000$

0.005631000

0.006905000

$-1.204997000$

$-1.185597000$

0.045415000

1.257036000

1.238033000

0.004063000

0.017921000

0.030231000

0.027793000

0.013119000

0.001251000

0.013013000

0.037285000

0.045675000

0.030114000

0.005897000

$-0.002908000$

0.017082000

$-2.162460000$

$-2.121247000$

0.059802000

2. 206442000

2.179775000

0.020211000

0.041581000

0.036931000

0.010602000 
PgHgHDz (blue-N(4)H) Gaussian (HF/SSD)

\begin{tabular}{|c|c|}
\hline $\mathrm{Hg}$ & 1.566715000 \\
\hline $\mathrm{S}$ & -0.703672000 \\
\hline $\mathrm{N}$ & -0.128789000 \\
\hline $\mathrm{N}$ & -1.338595000 \\
\hline $\mathrm{C}$ & -1.778182000 \\
\hline $\mathrm{N}$ & -3.034838000 \\
\hline $\mathrm{N}$ & -3.935675000 \\
\hline $\mathrm{C}$ & 3.615132000 \\
\hline $\mathrm{C}$ & 4.400083000 \\
\hline C & 5.756889000 \\
\hline $\mathrm{C}$ & 6.358066000 \\
\hline $\mathrm{C}$ & 5.595064000 \\
\hline $\mathrm{C}$ & 4.238458000 \\
\hline $\mathrm{C}$ & 0.236502000 \\
\hline $\mathrm{C}$ & 1.580578000 \\
\hline $\mathrm{C}$ & 2.014865000 \\
\hline $\mathrm{C}$ & 1.107449000 \\
\hline $\mathrm{C}$ & -0.238626000 \\
\hline $\mathrm{C}$ & -0.678204000 \\
\hline $\mathrm{C}$ & -5.304413000 \\
\hline $\mathrm{C}$ & -6.208321000 \\
\hline $\mathrm{C}$ & -7.574728000 \\
\hline $\mathrm{C}$ & -8.055376000 \\
\hline $\mathrm{C}$ & -7.145904000 \\
\hline $\mathrm{C}$ & -5.776871000 \\
\hline $\mathrm{H}$ & -3.624202000 \\
\hline $\mathrm{H}$ & 3.966023000 \\
\hline $\mathrm{H}$ & 6.336625000 \\
\hline $\mathrm{H}$ & 7.400631000 \\
\hline $\mathrm{H}$ & 6.049494000 \\
\hline $\mathrm{H}$ & 3.678109000 \\
\hline $\mathrm{H}$ & 2.274198000 \\
\hline $\mathrm{H}$ & 3.048126000 \\
\hline $\mathrm{H}$ & 1.440569000 \\
\hline $\mathrm{H}$ & -0.935225000 \\
\hline $\mathrm{H}$ & -1.704608000 \\
\hline $\mathrm{H}$ & -5.849020000 \\
\hline $\mathrm{H}$ & -8.257691000 \\
\hline $\mathrm{H}$ & -9.107077000 \\
\hline $\mathrm{H}$ & -7.500813000 \\
\hline $\mathrm{H}$ & -5.083063000 \\
\hline
\end{tabular}
$-1.205593000$
$-2.030798000$
1.042035000
0.771824000
$-0.575836000$
$-0.785798000$
0.198648000
$-0.761630000$
$-0.387188000$
$-0.091094000$
$-0.163596000$
$-0.534605000$
$-0.830847000$
2.427577000
2.709545000
4.033406000
5.071587000
4.783448000
3.465547000
$-0.100418000$
0.955782000
0.699437000
$-0.607913000$
$-1.655849000$
$-1.414431000$
1.142472000
$-0.325397000$
0.189876000
0.061507000
$-0.596292000$
$-1.117634000$
1.908408000
4.246950000
6.090060000
5.581342000
3.240108000
1.966279000
1.518055000
$-0.804807000$
$-2.666507000$
$-2.218750000$

$-0.104596000$

$-0.392141000$

$-0.117735000$

$-0.130558000$

$-0.174845000$

$-0.091348000$

0.047706000

0.117932000

$-0.985895000$

$-0.838845000$

0.417976000

1. 525217000

1.375303000

$-0.090076000$

0.145696000

0.189115000

$-0.013173000$

$-0.260957000$

- 0.300135000

0.124583000

0.278832000

0.358900000

0.286836000

0.133129000

0.051163000

0.096774000

$-1.965657000$

$-1.696869000$

0.531604000

2.495208000

2. 244669000

0.295646000

0.375450000

0.015959000

$-0.425407000$

- 0.498422000

0.336557000

0.477257000

0.348608000

0.076196000

$-0.066665000$
12.052366000 10.097220000 11.921998000 10.963903000 10.142211000 9.260527000 9.357651000 13.685160000 13.917406000
0.052306000

1.131603000

2.308262000

3.047079000

2.695959000

3.640731000

4.794356000

$-1.143679000$

$-1.492606000$ 


$\begin{array}{lr}\mathrm{C} & -1.039705000 \\ \mathrm{C} & -0.055302000 \\ \mathrm{C} & 1.278488000 \\ \mathrm{C} & 1.627834000 \\ \mathrm{C} & -1.251238000 \\ \mathrm{C} & -1.651359000 \\ \mathrm{C} & -2.713160000 \\ \mathrm{C} & -3.410480000 \\ \mathrm{C} & -3.036015000 \\ \mathrm{C} & -1.977384000 \\ \mathrm{C} & 1.761333000 \\ \mathrm{C} & 1.102116000 \\ \mathrm{C} & 1.518624000 \\ \mathrm{C} & 2.602088000 \\ \mathrm{C} & 3.264192000 \\ \mathrm{C} & 2.852645000 \\ \mathrm{H} & 0.101591000 \\ \mathrm{H} & -1.479043000 \\ \mathrm{H} & -2.083426000 \\ \mathrm{H} & -0.327119000 \\ \mathrm{H} & 2.053780000 \\ \mathrm{H} & 2.675932000 \\ \mathrm{H} & -1.096388000 \\ \mathrm{H} & -2.997111000 \\ \mathrm{H} & -4.242156000 \\ \mathrm{H} & -3.584948000 \\ \mathrm{H} & -1.747781000 \\ \mathrm{H} & 0.264277000 \\ \mathrm{H} & 1.001183000 \\ \mathrm{H} & 2.934733000 \\ \mathrm{H} & 4.112706000 \\ \mathrm{H} & 3.359022000\end{array}$

15.029816000

15.920664000

15.694238000

14.581627000

12.578803000

13.781543000

14.519766000

14.083927000

12.885402000

12.130865000

8.490270000

8.547085000

7.742719000

6.877868000

6.819784000

7.613415000

10.810826000

13.236311000

15.198973000

16.789062000

16.384547000

14.423494000

14.127346000

15.449469000

14.665758000

12.523948000

11.176829000

9.234595000

7.792064000

6.252259000

6.147797000

7.583360000
$-2.265018000$

$-2.695503000$

$-2.354718000$

$-1.584922000$

2. 874138000

2. 251717000

2. 755168000

3.887205000

4.500293000

4.003378000

5.778910000

7.019651000

8.074472000

7.906195000

6.671877000

5.610781000

4.035874000

$-1.165281000$

$-2.529542000$

$-3.294960000$

$-2.686819000$

$-1.331447000$

1.380815000

2. 263482000

4.281266000

5.369446000

4.479563000

7.127567000

9.031571000

8.732987000

6.544956000

4.648162000

\section{0}

\section{PhHgHDz (blue-N (2) H)}

\section{Gaussian (ADF/B3LYP)}

$\begin{array}{lr}\mathrm{Hg} & 1.587563000 \\ \mathrm{~S} & -0.642655000 \\ \mathrm{~N} & -0.016644000 \\ \mathrm{~N} & -1.324657000 \\ \mathrm{C} & -1.741355000 \\ \mathrm{~N} & -3.128798000 \\ \mathrm{~N} & -3.881527000 \\ \mathrm{C} & 3.682674000 \\ \mathrm{C} & 4.240622000 \\ \mathrm{C} & 5.646111000 \\ \mathrm{C} & 6.510591000 \\ \mathrm{C} & 5.965852000 \\ \mathrm{C} & 4.561203000 \\ \mathrm{C} & 0.179414000 \\ \mathrm{C} & 1.438616000 \\ \mathrm{C} & 1.722627000 \\ \mathrm{C} & 0.766577000 \\ \mathrm{C} & -0.476331000 \\ \mathrm{C} & -0.774483000 \\ \mathrm{C} & -5.289550000 \\ \mathrm{C} & -6.106079000 \\ \mathrm{C} & -7.519895000 \\ \mathrm{C} & -8.123157000 \\ \mathrm{C} & -7.305835000 \\ \mathrm{C} & -5.893360000 \\ \mathrm{H} & -2.105258000 \\ \mathrm{H} & 3.595163000 \\ \mathrm{H} & \end{array}$

$-0.047806000$

$-0.208700000$

0.149630000

0.063954000

$-0.057297000$

$-0.072168000$

0.161249000

$-0.004995000$

$-0.682390000$

$-0.665347000$

0.032109000

0.709649000

0.688948000

0.078080000

0.561602000

0.528161000

0.014037000

$-0.480722000$

$-0.462124000$

0.106218000

0.377059000

0.348619000

0.050801000

$-0.218774000$

$-0.194252000$

0.168531000

$-1.225471000$ 


$\begin{array}{ll}\mathrm{H} & 6.057389000 \\ \mathrm{H} & 7.588925000 \\ \mathrm{H} & 6.623795000 \\ \mathrm{H} & 4.163567000 \\ \mathrm{H} & 2.160046000 \\ \mathrm{H} & 2.682399000 \\ \mathrm{H} & 0.989002000 \\ \mathrm{H} & -1.208525000 \\ \mathrm{H} & -1.710935000 \\ \mathrm{H} & -5.620056000 \\ \mathrm{H} & -8.143814000 \\ \mathrm{H} & -9.212025000 \\ \mathrm{H} & -7.771732000 \\ \mathrm{H} & -5.254516000\end{array}$

1.541558000
-0.033815000
-2.029769000
-2.453435000
2.070619000
4.522279000
6.158911000
5.299905000
2.870386000
1.857023000
1.660318000
-0.560379000
-2.570935000
-2.363953000

$-1.191376000$

1. 246161000

1.209573000

0.975169000

0.908518000

$-0.007572000$

$-0.898151000$

$-0.908877000$

0.605333000

0.555978000

0.028709000

$-0.446372000$

$-0.396927000$

21 PgHgHDz (blue-N (2)H) Gaussian (HF/SSD)

$\begin{array}{lrrr}\mathrm{Hg} & 1.696171000 & -0.155927000 & 0.079760000 \\ \mathrm{~S} & -0.446515000 & -1.793201000 & -1.162984000 \\ \mathrm{~N} & -0.032751000 & 1.070101000 & 0.128778000 \\ \mathrm{~N} & -1.305476000 & 0.523852000 & -0.038641000 \\ \mathrm{C} & -1.577701000 & -0.648673000 & -0.550807000 \\ \mathrm{~N} & -2.967067000 & -1.010472000 & -0.586655000 \\ \mathrm{~N} & -3.673953000 & -0.537050000 & 0.319604000 \\ \mathrm{C} & 3.573611000 & -1.072454000 & 0.180039000 \\ \mathrm{C} & 4.710798000 & -0.388836000 & 0.641253000 \\ \mathrm{C} & 5.955235000 & -1.019241000 & 0.699689000 \\ \mathrm{C} & 6.086729000 & -2.348550000 & 0.298228000 \\ \mathrm{C} & 4.967447000 & -3.042425000 & -0.162145000 \\ \mathrm{C} & 3.724430000 & -2.410219000 & -0.220624000 \\ \mathrm{C} & 0.000928000 & 2.473117000 & -0.061743000 \\ \mathrm{C} & 1.024946000 & 3.207197000 & 0.558864000 \\ \mathrm{C} & 1.118635000 & 4.581794000 & 0.383392000 \\ \mathrm{C} & 0.182929000 & 5.267234000 & -0.396942000 \\ \mathrm{C} & -0.841087000 & 4.545801000 & -1.004367000 \\ \mathrm{C} & -0.933742000 & 3.161598000 & -0.848368000 \\ \mathrm{C} & -5.061421000 & -0.857996000 & 0.316261000 \\ \mathrm{C} & -5.815300000 & -0.288445000 & 1.340198000 \\ \mathrm{C} & -7.184016000 & -0.542056000 & 1.413992000 \\ \mathrm{C} & -7.786436000 & -1.366745000 & 0.464652000 \\ \mathrm{C} & -7.022337000 & -1.939709000 & -0.558413000 \\ \mathrm{C} & -5.657867000 & -1.689723000 & -0.638520000 \\ \mathrm{H} & -2.057592000 & 1.024650000 & 0.397312000 \\ \mathrm{H} & 4.639387000 & 0.634668000 & 0.955899000 \\ \mathrm{H} & 6.810903000 & -0.477370000 & 1.054370000 \\ \mathrm{H} & 7.042362000 & -2.833940000 & 0.342654000 \\ \mathrm{H} & 5.059177000 & -4.065064000 & -0.473731000 \\ \mathrm{H} & 2.879176000 & -2.964902000 & -0.579771000 \\ \mathrm{H} & 1.728570000 & 2.700479000 & 1.191092000 \\ \mathrm{H} & 1.911006000 & 5.120424000 & 0.865925000 \\ \mathrm{H} & 0.253391000 & 6.328891000 & -0.524756000 \\ \mathrm{H} & -1.564385000 & 5.052128000 & -1.613968000 \\ \mathrm{H} & -1.706488000 & 2.625993000 & -1.362008000 \\ \mathrm{H} & -5.328032000 & 0.337435000 & 2.060385000 \\ \mathrm{H} & -7.768296000 & -0.106111000 & 2.199129000 \\ \mathrm{H} & -8.838465000 & -1.565540000 & 0.519424000 \\ \mathrm{H} & -7.490023000 & -2.576036000 & -1.282732000 \\ \mathrm{H} & -5.060120000 & -2.125391000 & -1.411726000 \\ & & & \end{array}$

PhHgHDz (blue-S (1) H) ADF (PW91)

$\mathrm{Hg}$

0.794583000

12.442989000

0.332524000 


\begin{tabular}{|c|c|}
\hline $\mathrm{S}$ & 3.016523000 \\
\hline $\mathrm{N}$ & 0.274870000 \\
\hline $\mathrm{N}$ & 0.844997000 \\
\hline $\mathrm{C}$ & 1.983322000 \\
\hline $\mathrm{N}$ & 2.389363000 \\
\hline $\mathrm{N}$ & 3.425754000 \\
\hline $\mathrm{C}$ & 0.695773000 \\
\hline C & -0.569631000 \\
\hline C & -0.708483000 \\
\hline $\mathrm{C}$ & 0.413516000 \\
\hline C & 1.676100000 \\
\hline $\mathrm{C}$ & 1.820775000 \\
\hline $\mathrm{C}$ & -0.938042000 \\
\hline C & -1.617310000 \\
\hline C & -2.804888000 \\
\hline C & -3.336770000 \\
\hline C & -2.662176000 \\
\hline $\mathrm{C}$ & -1.476946000 \\
\hline C & 3.825095000 \\
\hline C & 4.849113000 \\
\hline C & 5.332812000 \\
\hline C & 4.809092000 \\
\hline $\mathrm{C}$ & 3.790314000 \\
\hline C & 3.296344000 \\
\hline $\mathrm{H}$ & -1.459986000 \\
\hline $\mathrm{H}$ & -1.697445000 \\
\hline $\mathrm{H}$ & 0.303110000 \\
\hline $\mathrm{H}$ & 2.554678000 \\
\hline $\mathrm{H}$ & 2.815619000 \\
\hline $\mathrm{H}$ & -1.208829000 \\
\hline $\mathrm{H}$ & -3.309510000 \\
\hline $\mathrm{H}$ & -4.261667000 \\
\hline $\mathrm{H}$ & -3.064874000 \\
\hline $\mathrm{H}$ & -0.949913000 \\
\hline $\mathrm{H}$ & 5.252585000 \\
\hline $\mathrm{H}$ & 6.126488000 \\
\hline $\mathrm{H}$ & 5.195784000 \\
\hline $\mathrm{H}$ & 3.387404000 \\
\hline $\mathrm{H}$ & 2.511877000 \\
\hline $\mathrm{H}$ & 3.27313700 \\
\hline
\end{tabular}

10.916833000

11.816220000

10.868470000

10.313430000

9.279622000

8.626293000

13.479322000

13.699848000

14.509990000

15.111598000

14.887934000

14.072658000

12.316463000

13.336674000

13.863087000

13.387343000

12.374435000

11.838446000

7.630505000

6.767139000

5.780394000

5.651784000

6.513635000

7.494727000

13.249484000

14.677170000

15.756968000

15.356481000

13.916230000

13.737719000

14.655755000

13.803959000

11.995309000

11.051596000

6.890983000

5.113360000

4.890095000

6.420608000

8.177442000

9.652933000
1. 345682000

2. 371818000

3.069393000

2.735478000

3. 522430000

3.150861000

$-1.476811000$

$-2.039784000$

$-3.169462000$

$-3.740359000$

$-3.189681000$

$-2.063391000$

2. 905380000

2. 220188000

2. 719936000

3.918838000

4.606498000

4.113681000

4.069357000

3.649324000

4.505262000

5.793078000

6.218004000

5.368065000

$-1.600302000$

$-3.594736000$

$-4.610860000$

$-3.632131000$

$-1.645400000$

1. 291370000

2.169224000

4.314317000

5.544907000

4.646393000

2.645173000

4.170942000

6.468737000

7. 226516000

5.687303000

0.904315000

\section{PhHgHDz (blue-S(1)H) Gaussian (ADF/B3LYP)}

$\begin{array}{lr}\mathrm{Hg} & 1.761686000 \\ \mathrm{~S} & -0.491787000 \\ \mathrm{~N} & -0.026759000 \\ \mathrm{~N} & -1.301850000 \\ \mathrm{C} & -1.685555000 \\ \mathrm{~N} & -3.026979000 \\ \mathrm{~N} & -3.945871000 \\ \mathrm{C} & 3.616944000 \\ \mathrm{C} & 4.804926000 \\ \mathrm{C} & 6.054286000 \\ \mathrm{C} & 6.130850000 \\ \mathrm{C} & 4.954872000 \\ \mathrm{C} & 3.703412000 \\ \mathrm{C} & 0.075281000 \\ \mathrm{C} & 1.359622000 \\ \mathrm{C} & 1.498045000 \\ \mathrm{C} & 0.358623000 \\ \mathrm{C} & -0.922681000 \\ \mathrm{C} & -1.075155000\end{array}$

-0.117149000
-2.092834000
1.078860000
0.618699000
-0.629044000
-1.074437000
-0.156331000
-1.091801000
-0.364463000
-1.038504000
-2.442922000
-3.173952000
-2.503740000
2.519796000
3.130085000
4.541648000
5.365313000
4.755376000
3.346468000

$-0.041746000$

$-0.464862000$

$-0.197708000$

$-0.172285000$

$-0.227025000$

$-0.154136000$

0.026574000

0.125302000

0.453739000

0.565859000

0.349640000

0.021857000

$-0.089076000$

$-0.124982000$

$-0.269363000$

$-0.191967000$

0.022990000

0.156783000

0.085010000 


$\begin{array}{ll}\mathrm{C} & -5.286790000 \\ \mathrm{C} & -6.295431000 \\ \mathrm{C} & -7.658861000 \\ \mathrm{C} & -8.020736000 \\ \mathrm{C} & -7.012257000 \\ \mathrm{C} & -5.648128000 \\ \mathrm{H} & 4.767262000 \\ \mathrm{H} & 6.954377000 \\ \mathrm{H} & 7.090829000 \\ \mathrm{H} & 5.007924000 \\ \mathrm{H} & 2.810420000 \\ \mathrm{H} & 2.246552000 \\ \mathrm{H} & 2.488211000 \\ \mathrm{H} & 0.463918000 \\ \mathrm{H} & -1.807861000 \\ \mathrm{H} & -2.052778000 \\ \mathrm{H} & -5.987862000 \\ \mathrm{H} & -8.428748000 \\ \mathrm{H} & -9.070053000 \\ \mathrm{H} & -7.292987000 \\ \mathrm{H} & -4.867497000 \\ \mathrm{H} & -0.985424000\end{array}$
$-0.674440000$
0.309108000
$-0.078942000$
$-1.447029000$
$-2.427756000$
$-2.051662000$
0.714311000
$-0.471926000$
$-2.958805000$
$-4.252744000$
$-3.080048000$
2.518252000
4.989715000
6.451184000
5.376466000
2.878272000
1. 349844000
0.673776000
$-1.747122000$
$-3.476481000$
$-2.787055000$
$-2.849131000$

0.090651000
0.334620000
0.419177000
0.257619000
0.009141000
-0.076292000
0.624122000
0.818410000
0.434993000
-0.146254000
-0.342295000
-0.461500000
-0.307351000
0.080095000
0.318790000
0.184091000
0.451836000
0.606623000
0.320605000
-0.119183000
-0.271327000
0.586594000

PgHgHDz (blue-S(1)H) Gaussian (HF/SSD)

$\begin{array}{lr}\mathrm{Hg} & 0.313086000 \\ \mathrm{~S} & 2.235886000 \\ \mathrm{~N} & -0.427241000 \\ \mathrm{~N} & 0.231245000 \\ \mathrm{C} & 1.309656000 \\ \mathrm{~N} & 1.900845000 \\ \mathrm{~N} & 1.575344000 \\ \mathrm{C} & 0.888327000 \\ \mathrm{C} & 0.270117000 \\ \mathrm{C} & 0.657816000 \\ \mathrm{C} & 1.671774000 \\ \mathrm{C} & 2.295605000 \\ \mathrm{C} & 1.907529000 \\ \mathrm{C} & -1.647257000 \\ \mathrm{C} & -2.586265000 \\ \mathrm{C} & -3.773373000 \\ \mathrm{C} & -4.053102000 \\ \mathrm{C} & -3.124838000 \\ \mathrm{C} & -1.933925000 \\ \mathrm{C} & 2.163288000 \\ \mathrm{C} & 1.845180000 \\ \mathrm{C} & 2.369414000 \\ \mathrm{C} & 3.207540000 \\ \mathrm{C} & 3.519947000 \\ \mathrm{C} & 3.000774000 \\ \mathrm{H} & -0.512426000 \\ \mathrm{H} & 0.172788000 \\ \mathrm{H} & 1.969735000 \\ \mathrm{H} & 3.075548000 \\ \mathrm{H} & 2.402605000 \\ \mathrm{H} & -2.417875000 \\ \mathrm{H} & -4.478094000 \\ \mathrm{H} & -4.967525000 \\ \mathrm{H} & -3.326429000 \\ \mathrm{H} & -1.232583000 \\ \mathrm{H} & 1.196099000 \\ \mathrm{H} & 2.125515000 \\ & \end{array}$

12.869512000

0.491617000

10.522958000

11.917833000

1.157008000

11.040700000

10.433379000

9.511158000

9.592700000

13.941987000

15.157923000

15.871831000

15.382339000

14.175986000

13.464046000

12.372756000

13.025614000

13.515814000

13.352969000

12.686128000

12.198893000

8.644786000

8.815240000

7.945058000

6.903776000

6.733004000

7.598609000

15.558958000

16.798878000

15.929980000

13.791694000

12.538116000

13.129999000

2. 219909000

2.991782000

2.744594000

3.652603000

4.849330000

$-1.205414000$

$-1.541500000$

$-2.676683000$

$-3.499994000$

$-3.181537000$

$-2.045472000$

2.802353000

1.995886000

2.536681000

3.891474000

4.694075000

4.163834000

5.741996000

7.087718000

8.043313000

7.647293000

6.294248000

5.338020000

$-0.926261000$

$-2.914091000$

$-4.372758000$

$-3.810064000$

$-1.824278000$

0.940140000

1.896188000

14.009811000

13.725637000

12.545349000

11.684893000

9.620808000

8.077209000

4.308465000

5.738497000

4.784452000

7.365670000

9.078637000 
